\title{
Recent progress in nanoimprint technology and its applications
}

\author{
L Jay Guo \\ Department of Electrical Engineering and Computer Science, The University of Michigan, \\ Ann Arbor, MI 48109, USA \\ E-mail: guo@umich.edu
}

Received 7 April 2004

Published 12 May 2004

Online at stacks.iop.org/JPhysD/37/R123

DOI: $10.1088 / 0022-3727 / 37 / 11 / R 01$

\begin{abstract}
Nanoimprint is an emerging lithographic technology that promises high-throughput patterning of nanostructures. Based on the mechanical embossing principle, nanoimprint technique can achieve pattern resolutions beyond the limitations set by the light diffractions or beam scatterings in other conventional techniques. This article reviews the basic principles of nanoimprint technology and some of the recent progress in this field. It also explores a few alternative approaches that are related to nanoimprint as well as additive approaches for patterning polymer structures. Nanoimprint technology can not only create resist patterns as in lithography but can also imprint functional device structures in polymers. This property is exploited in several non-traditional microelectronic applications in the areas of photonics and biotechnology.
\end{abstract}

(Some figures in this article are in colour only in the electronic version)

\section{Introduction}

The ability to replicate patterns at the micro- to the nanoscale is of crucial importance to the advance of micro- and nanotechnologies and the study of nanosciences. Critical issues such as resolution, reliability, speed, and overlay accuracy all need to be considered in developing new lithography methodologies. The primary driver for reliable and high-throughput nanolithography is the ability to make ever-shrinking transistors on an IC chip. With its unrelenting effort to make faster and more powerful microchips, the semiconductor industry is pushing to reduce the transistor gate length down to $22 \mathrm{~nm}$ by 2010 . The challenges of following such a roadmap are multi-fold, and one of the biggest challenges is believed to be lithography - the technique used to create micro- to nanoscale patterns in a resist material. Because of the planar fabrication technology employed by the semiconductor industry, integrated circuits are built by stacking one layer of circuit elements on top of another. Each layer is fabricated by a sequence of well-characterized processes. Lithography is used over and over again to create desired patterns during all these processes, and as such the cost of lithography in IC production can amount to $35 \%$ of total chip cost. Considerable industrial effort has been devoted to the leading-edge optical methods and the so-called next generation lithography (NGL) techniques, exposing resist material with energetic beams from extreme UV, electron-beam, ion-beam or x-ray sources. Not only are there a lot of issues that are yet to be solved in DUV photolithography and various NGL methods, the cost of a single NGL tool could also exceed $\$ 50$ million in the next few years-a formidable price tag for most potential users. On the other hand, there are many nontraditional microelectronic applications that require nanoscale features and demand low-cost nanopatterning technologies for gaining a competitive edge.

In recent years, researchers have investigated a number of alternative and potentially low-cost methods: most notably, microcontact printing $(\mu \mathrm{CP})$, nanoimprint technology, AFM lithography and dip-pen lithography. Since the mid1990s, nanoimprint lithography (NIL), initially proposed and developed by the Chou group [1,2], has emerged as one of the most promising technologies for high-throughput 
nanoscale patterning. In this method and its variants, such as step-and-flash imprint lithography (S-FIL), which was developed by Willson's group, pattern replication is done nontraditionally by deforming mechanically the resist materials, which makes them completely free from the resolutionlimiting factors such as light diffraction and beam scattering that are often inherent with the more traditional approaches. MIT's Technology Review has recently put NIL as one of 10 emerging technologies that are likely to change the world [3].

Imprinting-based lithography also presents many new challenges to researchers. Investigations made in recent years by various groups, as well as our own, have identified many critical issues that need to be addressed for the further progress of this technology, such as its limitations in handling complex patterns with varied feature density, patterning over topographies and pattern alignment, to name an important few. There is also great demand for new material systems with properties more suitable for the imprint application, with off-the-shelf commercial polymers having become inadequate to satisfy the special requirements of nanoimprinting. In addition, new techniques are needed for many non-traditional microelectronics applications. This article reviews some of the recent developments in the area of imprint technology and its various applications. The readers are referred to a recent book, 'Alternative Lithography' [4], which includes chapters dedicated to the discussion of various aspects of NIL written by several experts in the fields. To minimize possible overlap and repetition, this review describes other new developments in this field, including much recent work carried out at the University of Michigan.

The principle of nanoimprint lithography is quite simple. As shown in figure 1(a), NIL uses a hard mould that contains nanoscale features defined on its surface to emboss into polymer material cast on the wafer substrate under controlled temperature and pressure conditions, thereby creating a thickness contrast in the polymer material, which can be further transferred through the resist layer via an $\mathrm{O}_{2}$ plasmabased anisotropic etching process. Nanoimprint lithography has the capability of patterning sub $10 \mathrm{~nm}$ features [5], yet it only entails simple equipment and easy processing. This is the key reason why NIL has attracted wide attention within only a few years after its inception.
NIL (Chou)
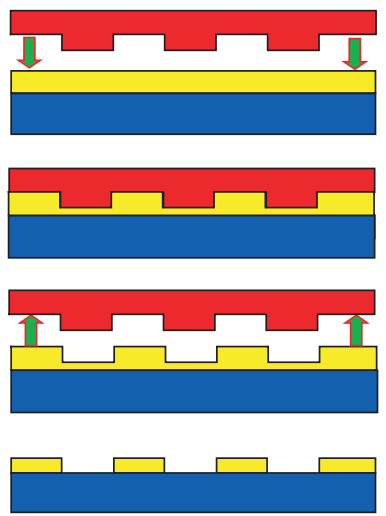

(a)
S-FIL (Willson)
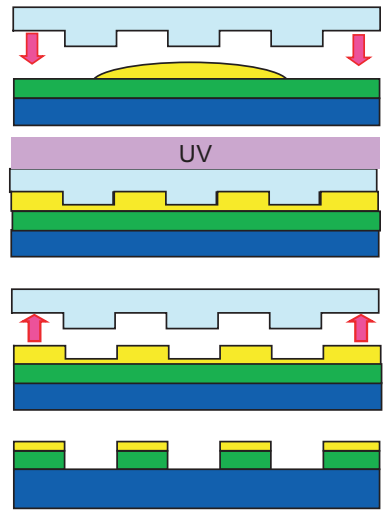

(b)
Figure 1. Schematic of (a) NIL and (b) S-FIL.
S-FIL is another great success as a mechanical printing method (figure 1(b)) [6]. In the S-FIL process, the substrate is first coated with an organic transfer layer; then a surfacetreated, transparent template with surface relief patterns is brought close and aligned to the coated substrate. Once in the proximity, a drop of low viscosity, photopolymerizable organosilicon solution is introduced into the gap between the template and the substrate. The organosilicon fluid spreads out and fills the gap under capillary action. Next the template is pressed against the substrate to close the gap, and the assembly is irradiated with UV light, which cures the photopolymer to make it a solidified and a silicon-rich replica of the template. Significant efforts from both academia and industry have been put in S-FIL research and development [7], template fabrication methods [8,9], and defect analysis $[9,10]$. Because of its ability to pattern at room temperature and at low pressure, the template can be stepped to pattern the whole wafer area as in a stepper lithography tool. These imprinting-based lithography technologies, especially S-FIL, are entering the competition among NGL methodologies for future semiconductor IC manufacturing and are emerging as strong contenders. For recent development in S-FIL technology for IC fabrication, the readers can refer to a paper by Resnick et al [11].

\section{Nanoimprint mould}

The mould in the nanoimprint technique plays the same role as the photomask in photolithography. Both NIL and S-FIL utilize hard moulds to replicate patterns, which distinguishes them from the widely studied $\mu \mathrm{CP}$ technique, which uses a soft and flexible poly(dimethylsiloxane) (PDMS) stamp. The hard features on NIL moulds and S-FIL templates can allow imprinting of features in polymer materials with resolutions below $10 \mathrm{~nm}$, which is not possible to achieve by $\mu \mathrm{CP}$, using a soft stamp. Hard moulds have mostly been made of $\mathrm{Si}$ or $\mathrm{SiO}_{2}$ for nanoimprinting, while it is also possible to use a metal as the mould material.

Considerations for selecting mould materials include the hardness, the compatibility with traditional microfabrication processing and the thermal expansion coefficient, to name an important few. Li has examined the relative hardness of various materials suitable for imprinting moulds, including $\mathrm{Si}, \mathrm{SiO}_{2}$, $\mathrm{SiC}$, silicon nitride and sapphire [12]. Taniguchi et al [13, 14] have investigated diamond as a potential mould material for NIL. Pang et al [15] have demonstrated nanoprinting of $\mathrm{Al}$ using a $\mathrm{SiC}$ mould. On the other hand, work by many groups have shown that $\mathrm{Si}$ and $\mathrm{SiO}_{2}$ have sufficient hardness and durability for the nanoimprint application. The thermal expansion coefficient is especially important in thermal NIL, where a temperature over $100^{\circ} \mathrm{C}$ is typically required in the imprinting step. A thermal mismatch between the mould and the substrate could result in pattern distortions or a stress build-up during the cooling cycle, which would affect the pattern fidelity. In this regard, a $\mathrm{Si}$ mould and $\mathrm{Si}$ substrate make a very good pair for the NIL process.

One would normally imagine that the nanoscale protrusion features on the mould is a weak point of NIL. But this is usually not the case. The reason is that in imprint lithography, mould durability is maintained by choosing the proper polymer resist 
layer thickness such that the mould protrusion will not contact the hard substrate directly. In other words, a thin viscous polymer fluid exists between the mould protrusions and the substrate surface and acts as a 'soft cushion', which effectively protects the nanofeatures on the mould. The trade-off of this is the existence of a residual resist layer that is present in the recessed regions, which has to be removed by a separate plasma etching step before pattern definition can be completed.

A mould used for imprint lithography typically has a high density of nanoscale protrusion features on its surface, which effectively increases the total surface area that contacts the imprinted polymer and therefore leads to a strong tendency of adhesion of the imprinted polymer to the mould. This effect can be seen easily from the sticking of the resist material on a mould that has no special treatment. The solution to this problem is to apply a low surface tension coating to the mould to reduce its surface energy. This can be done by plasma deposition of fluropolymers [16] or by using a monolayer of perfluorosilane surfactant molecules. The latter technique is the most widely adopted method. Surfactant molecules, such as $1 \mathrm{H}, 1 \mathrm{H}, 2 \mathrm{H}, 2 \mathrm{H}$-perfluorodecyl-trichlorosilane (FDTS) can be deposited on the mould surface either as a liquid phase or as a vapour phase through a silanization process (figure 2). Silanization of oxidized silicon with a $\mathrm{RSiCl}_{3}$ precursor begins with hydrolysis of the polar headgroup, which converts the $\mathrm{Si}-\mathrm{Cl}$ bonds to $\mathrm{Si}-\mathrm{OH}$ (silanol) groups $[17,18]$. The silanol groups generated, which are strongly attracted to the hydrophilic surface of oxided silicon, condense and react with the hydroxyl group on the surface as well as other monomer silanol group to form networks of covalent siloxane bonds, $\mathrm{Si}-\mathrm{O}-\mathrm{Si}$. Such covalent bonding makes the surfactant coating layer chemically and thermally stable. Vapour phase coating has been shown to provide better imprint results for nanoscale features because it avoids the difficulty of liquid wetting of the nanoscale trench features on the mould [19].

A more important issue in terms of mould durability is actually related to the wearing and stability of this surfactant coating layer. This could be a critical issue for the acceptance of imprint-based technology in industrial applications. The

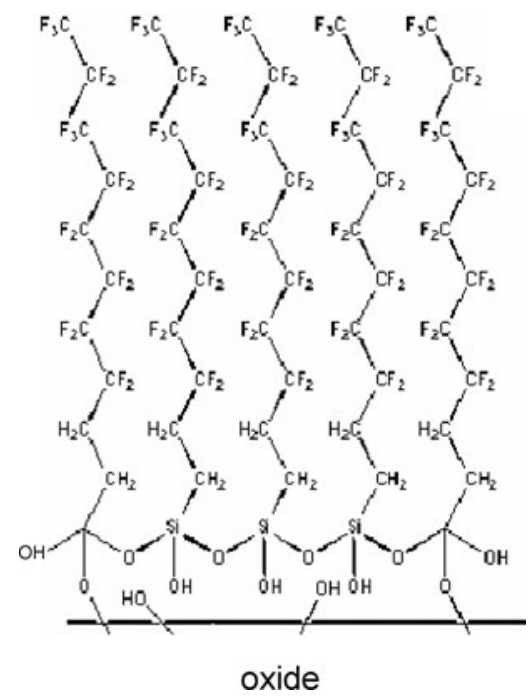

Figure 2. Formation of a monolayer of FDTS molecules on $\mathrm{SiO}_{2}$ to create a low-energy surface. testing method has so far been making multiple imprints and then comparing the imprinted patterns in order to deduce if the surfactant coating has been damaged. Taniguchi et al have measured the adhesive force between moulds treated with different surfactant molecules and photocurable polymers, but this has shown no direct correlation between the adhesive force and the durability of the surfactant coating [20]. There has been no analytical method of looking at the process at a microscopic level, and therefore the behaviour of the surfactant molecules during imprinting has not been investigated. Many surface analysis methods, such as AFM, XPS, ellipsometry, etc. cannot be applied in this situation because these surfactant molecules are buried beneath the polymer resist layer. A nondestructive characterization technique is highly desirable for this purpose. A possible candidate could be a special nonlinear optical (NLO) method, the sum frequency generation (SFG) technique, for investigating the behaviour of surfactant molecules during different steps of the imprint process. SFG is a powerful and versatile in situ surface probe that not only permits identification of surface molecular species but also provides information about surface structures [21]. SFG is a process in which two input beams at frequencies $\omega_{1}$ and $\omega_{2}$ mix in a medium and generate an output beam at the sum frequency, $\omega=\omega_{1}+\omega_{2}$. As a second-order NLO process, it is forbidden under the electric-dipole approximation in media that posses inversion symmetry. At surfaces or interfaces, on the other hand, because the inversion symmetry is broken, SFG is allowed naturally and therefore can be used as an effective surface analysis method.

Finally we should point out that the durability issue of the surface coating on moulds can be alleviated if the mould itself is made of a material that has low surface energy. Recently, it has been demonstrated that amorphous fluoropolymers, such as Tyflon $\mathrm{AF}\left(T_{\mathrm{g}}=240^{\circ} \mathrm{C}\right)$, can be used as an imprinting mould without any surface treatment. Mould fabrication can also be simplified by casting the fluoropolymer solutions over a prefabricated template and drying off the solvent [22] or by direct moulding or imprinting of this fluoropolymer at $350^{\circ} \mathrm{C}$ under a high pressure [23]. A flexible film mould can be obtained using these methods that can provide better conformal contact with the substrate to be patterned and reduce the pressure needed during the imprinting step.

\section{Nanoimprint resist}

Because imprint lithography makes a conformal replica of the surface relief patterns by mechanical embossing, the resist materials used in imprinting should be deformable easily under an applied pressure. S-FIL uses a very effective solution by utilizing a liquid resist that can be cured and solidified by UV exposure. The viscosity of the initial monomer liquid is chosen to be lower than 4 cps to allow a very low pressure printing. In NIL, typically a thermal plastic material is used as the imprint resist, and a suitable imprint temperature is chosen $70-80^{\circ} \mathrm{C}$ above the material's glass transition temperature $\left(T_{\mathrm{g}}\right)$. This choice can be explained by considering the typical deformation behaviour of a thermal plastic polymer as a function of the temperature (figure 3). At a temperature below $T_{\mathrm{g}}$, the major contribution to the deformation comes from the elongation of the atomic distance, and the deformation 


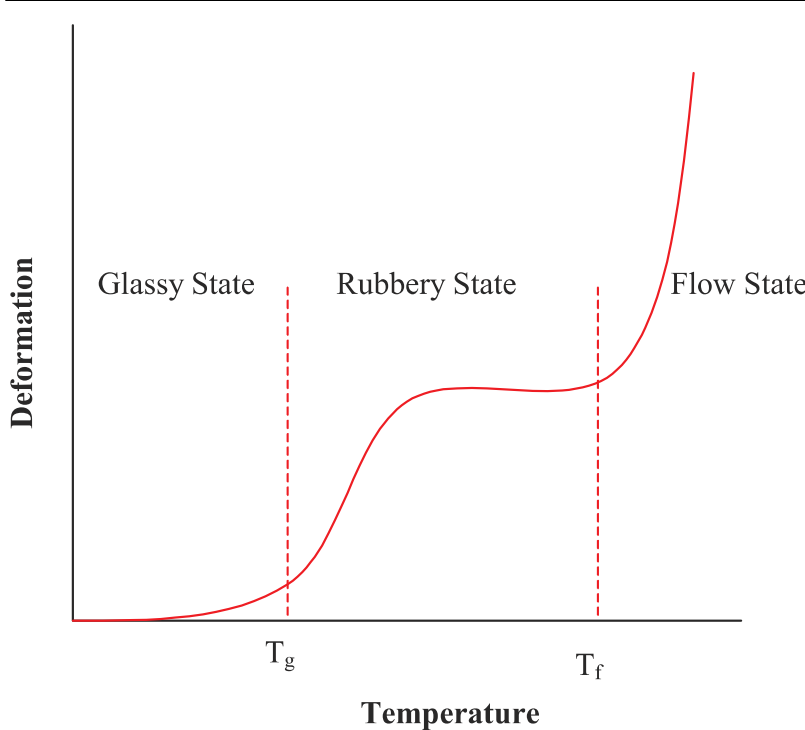

Figure 3. Typical deformation behaviour of thermal plastic polymers as a function of temperature.

is ideal elastic. The Young's modulus for glassy polymers just below $T_{\mathrm{g}}$ is approximately constant over a wide range of polymers $\left(3 \times 10^{9} \mathrm{~Pa}\right)$, and the magnitude of deformation is very small. Above $T_{\mathrm{g}}$, local motion of chain segments takes place and the modulus of the material drops by several orders of magnitude. However, the entire chains are still fixed by the temporary network of entanglements. A rubber-elastic plateau region exists beyond $T_{\mathrm{g}}$, where a relatively large deformation may occur due to extension of chain segments fixed between entanglement points. The modulus stays relatively constant in the rubbery state, and the deformation will recover after the force is released. Next is the rubbery flow region for linear amorphous polymers, but it does not occur for cross-linked polymers. Finally, with a further increase in temperature, the viscous liquid flow state is reached. In this regime motion of entire chains takes place and the polymer flows by chain sliding. The modulus and viscosity are further reduced in this region and the deformation is irreversible, which makes it the right temperature range for NIL patterning. It is perceived that a good imprinting result can be acquired when the imprinting temperature is set to be higher than the flow temperature $\left(T_{\mathrm{f}}\right)$ of the polymer. Empirically an optimal imprinting temperature is found $70-80^{\circ} \mathrm{C}$ above the $T_{\mathrm{g}}$ of the material used. In this temperature range, the viscosity of the polymer can be described by the following equation of Williams, Landel and Ferry:

$$
\log \eta(T)=\log \eta\left(T_{\mathrm{g}}\right)-\frac{C_{1}\left(T-T_{\mathrm{g}}\right)}{C_{2}+\left(T-T_{\mathrm{g}}\right)}
$$

where the values for $C_{1}$ and $C_{2}$ are 17.44 and 51.6, respectively. This equation describes the effect of temperature on viscosity for a large number of polymers. Since $T_{\mathrm{g}}$ is the onset temperature for molecular motion in polymers, factors that increase the energy required for molecular motion also increase $T_{\mathrm{g}}$; those that decrease the energy requirement lower $T_{\mathrm{g}}$ [24]. These considerations can be exploited in choosing the desired $T_{\mathrm{g}}$ for the imprint resist. Therefore factors such as intermolecular forces, intrachain steric hindrance (e.g. branching or crosslinking) and bulky and stiff side groups

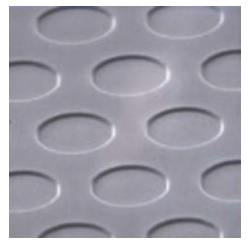

(a)

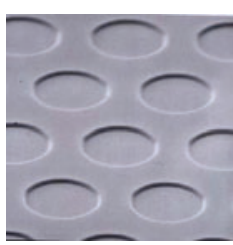

(b)

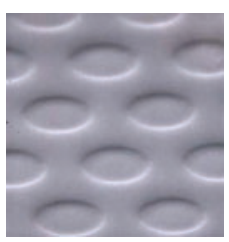

(c)
Figure 4. SEM micrograph of patterns imprinted in (a) poly(benzyl methacrylate) and (b) poly(cyclohexyl acrylate); (c) relaxation of poly(cyclohexyl acrylate) patterns 10 days after the imprinting.

can be used to increase $T_{\mathrm{g}}$, while flexible bonds and flexible side groups can decrease $T_{\mathrm{g}}$.

Often it is desirable to do patterning at a lower temperature for higher throughput and reducing the thermal expansion mismatch. From the above analysis, one can use materials that have a lower $T_{\mathrm{g}}$ as the NIL resist. Figure 4(a) shows the imprinted patterns in poly(benzyl methacrylate) $\left(M_{\mathrm{w}} \sim 70000, T_{\mathrm{g}}=54^{\circ} \mathrm{C}\right)$ at a temperature of $134^{\circ} \mathrm{C}$ and figure $4(b)$ the patterns imprinted in poly(cyclohexyl acrylate) $\left(M_{\mathrm{w}} \sim 150000 ; T_{\mathrm{g}}=19^{\circ} \mathrm{C}\right)$ at $99^{\circ} \mathrm{C}$. In both cases, the imprinting temperature was chosen to be $T_{\mathrm{g}}+80^{\circ} \mathrm{C}$ and the applied pressure was $50 \mathrm{~kg} \mathrm{~cm}^{-2}$, and very good pattern definition was obtained.

Another important criterion for a good NIL resist is that the imprinted pattern should maintain its mechanical integrity during mould-substrate separation as well as any subsequent pattern transfer steps. Although a low $T_{\mathrm{g}}$ material can be used for NIL for the sake of reducing the processing temperature, the imprinted patterns are also unstable and tend to deform at temperatures close to the imprinting temperature. Figure $4(c)$ shows the pattern relaxation at room temperature that was observed 10 days after the structures shown in $(b)$ were imprinted (in this case the resist has a $T_{\mathrm{g}}$ of $19^{\circ} \mathrm{C}$, less than the room temperature). From this demonstration, it can be inferred that thermal curable or thermosetting polymers could be excellent resist systems for NIL due to the possibility of low pressure imprinting and good mechanical integrity after cross-linking through thermal treatment.

An alternative method for making a thermal plastic material imprintable without resorting to a high temperature process is to dissolve the polymer in its monomer solution as demonstrated in [25], or to incorporate a certain amount of solvent in the polymer. Lee et al have developed a room temperature nanoimprint technique based on the latter strategy and using PDMS as the stamp material. By choosing a solvent that can be absorbed readily into the PDMS mould and evaporates through the mould into air, pattern replication with high fidelity can be obtained at room temperature and a pressure of less than $1 \mathrm{~N} \mathrm{~cm}^{-2}$ [26]. The drawback of this technique is the relatively long time $(\sim 10 \mathrm{~min})$ required for the solvent to evaporate completely through the elastomeric mould.

The viscosity not only has a temperature dependence but also depends strongly on the polymer's molecular weight, $M_{\mathrm{w}}$, relative to the so-called critical molecular weight of a given polymer, $M_{\mathrm{c}} . M_{\mathrm{c}}$ can be interpreted as the molecular weight at which a temporary network of entanglements spans macroscopic dimensions. Below $M_{\mathrm{c}}$ entanglements may be 
present, but their number is too small to lead to a sufficiently connected temporary network. Accordingly, the polymer melt has flow properties resembling those of a low molecular weight compound: the viscosity increases linearly with the molecular weight. Above $M_{\mathrm{c}}$ the segment length between entanglements appears to be constant according to experimental observation; the viscosity shows a stronger dependence on $M$ since the number of entanglements per chain has to increase with $M$ for a constant segment length (see sections 3 and 4 of [4]). If the Newtonian viscosity at the critical molecular weight is denoted by $\eta_{\mathrm{c}}$, the viscosity of a linear polymer in the flow state can be described by the following equations:

$$
\begin{aligned}
& \eta=\eta_{\mathrm{c}}\left(\frac{M_{\mathrm{w}}}{M_{\mathrm{c}}}\right) \quad\left(M_{\mathrm{w}}<M_{\mathrm{c}}, \text { unentangled molecules }\right) \\
& \eta=\eta_{\mathrm{c}}\left(\frac{M_{\mathrm{w}}}{M_{\mathrm{c}}}\right)^{3.4} \quad\left(M_{\mathrm{w}}>M_{\mathrm{c}}, \text { entangled molecules }\right)
\end{aligned}
$$

In practice, low molecular weight polymers with $M<M_{\mathrm{c}}$ can be imprinted at lower temperatures, lower pressures or within shorter times. However, the absence of a network of entanglements may lead to more brittle behaviour and could result in the fracture of the imprinted polymer features during the mould separation step. Therefore the choice of both $T_{\mathrm{g}}$ and $M_{\mathrm{w}}$ is important in pattern structural stability. On the other hand, this property can also be exploited in deciding the structures to be obtained in using the reverse imprinting technique to pattern over topographies (see sections 5 and 7). In addition the stress build-up during the thermal cycling of the NIL process also affects the pattern integrity during mould separation, and Hirai et al [27] have investigated this problem in detail. For more discussions on the material rheology issues related to nanoimprint technology, readers are referred to chapters 3 and 4 in [4].

Currently, a great opportunity exists for developing new materials and polymer formulations that are more suitable for nanoimprint applications. Up to now, researchers have mostly used commercial thermal plastic materials, e.g. poly(methylmethacrylate) (PMMA) and polystyrene (PS), as NIL resists $[5,28,29]$. Obviously, these materials are not optimized at all for the special requirements of the NIL process. One critical requirements of the candidate polymers used in imprintingbased lithography is that they should provide excellent mould releasing properties during the de-moulding process and at the same time should not compromise the adhesion of the mould to the substrate. Commercially available polymer materials can hardly satisfy these seemingly contradictory requirements. Although the mould surface is treated normally with a low surface energy surfactant, when imprinting high density or high aspect ratio patterns, the imprinted polymer tends to adhere to the mould, creating pattern defects that are not acceptable for many applications. Block copolymer materials that have dual surface properties through microphase segregation can be exploited to solve this problem. In addition, a higher dry etching resistance is highly desirable if the imprinted polymer pattern is to be used as a dry etching mask for further pattern transfer. Adding a Si-containing material in the polymer formulation could be an effective method. We can anticipate that many new materials with superior properties will be developed in the next few years to satisfy the needs of imprinting-based nanolithography.

\section{Combined nanoimprinting and photolithography}

Although NIL has proved to be very successful in nanopatterning, especially in replicating periodic nanoscale features with uniform sizes, it still has several limitations as a flexible lithographic technique. A general lithographic technique should be capable of producing both large and small features in various combinations and distributions-a typical requirement in micro- and nanofabrication processes. In NIL, protrusion features on the mould deform physically and displace the polymer. Larger features on the mould must displace more polymer material over larger distances. Thus, patterns with large features are much more difficult to imprint than nanopatterns. Moreover, NIL pattern defects or even failures in the form of incomplete pattern transfer can occur due to the high viscosity of the polymer melt and the mould pattern complexity.

Figure 5 illustrates several ways in which nanoimprinting could fail to produce faithful pattern replications. Consider a general situation where the mould consists of a combination of nanoscale patterns next to some large-scale patterns. If the mould and the substrate remain flat during imprinting, the mould relief patterns penetrate into the resist layer on the substrate in a parallel fashion as shown in figure 5(a). Because of the viscous polymer flow, the large-scale protrusions on the mould cannot penetrate the polymer film fully in a time frame that is practical for NIL. Not only will this cause incomplete polymer moulding of large-scale patterns [30], it will also affect the nanoscale patterns in close proximity to the large-scale features, yielding a shallower resist pattern than that on the mould [31].

On the other hand, if either the mould or the substrate can bend under a high pressure during imprinting, such that a conformal contact can be made at the polymer resist layer as shown in figure $5(b)$, the same feature height can be achieved for both large-scale and nanoscale patterns. However, due to mould bending, the nanoscale relief patterns penetrate deeper into the resist layer than with the large patterns, leading to a thinner residue layer in the nanoscale pattern region than that in the large pattern region [32]. This non-uniform thickness across the sample makes it difficult to determine the right amount of etching required to clear all patterned regions. Some

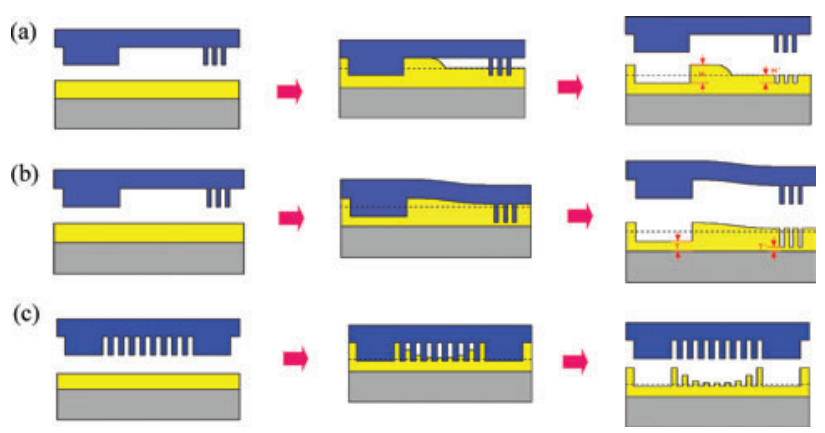

Figure 5. Several defect generation mechanisms in NIL that occur when mould contains complex patterns. The high viscosity prevents total displacement of the polymer under the large size patterns, which affects the imprint of nearby nanoscale patterns $(a)$ and $(b)$. Insufficient polymer flow over a large distance causes incomplete filling of nanoscale trenches $(c)$. Reprinted with permission from Elsevier [37]. 
patterns might be lost if sufficient residual layer is not removed, while over-removal can reduce the aspect ratio of the nanoscale resist feature.

Due to the mechanical moulding nature of the NIL, the displaced polymer melt must be accommodated by recessed regions on the mould, and so the location and size of recessed areas on the mould can affect the NIL result. Complex mask patterns will give rise to a random distribution of the recessed areas on the mould, and some patterns cannot be replicated fully in the imprinting process $[33,34]$. Figure $5(c)$ is an example where nanopatterns are next to the large structures on the mould. The displaced polymer cannot reach the centre of the nanopattern region, leaving the centre of the nanopatterns formed incompletely.

The aforementioned defect generations are related to the mould pattern itself and are inherent in the NIL process. In real applications, micro- and nanofabricated devices usually require a mixture of arbitrary patterns with various sizes. The previous approach to addressing the issue of replicating patterns of various sizes has been to use a mix-and-match method that is carried out in two separate steps in order to get around the pattern-related defect issues in NIL $[35,36]$. In this approach, not only is alignment needed for each patterning, its accuracy is also limited in the photolithography step. In addition, NIL requires a separate oxygen plasma etching process to remove the residual polymer layer in the imprinted regions, which adds to the process time. It is difficult to achieve a uniform residual layer thickness across the imprinted area when the patterns have various sizes and densities, making it a challenge to remove the right amount of residual polymers to complete the pattern definition.

In order to solve those problems, we developed a technique that integrates photolithography into NIL to enjoy the advantages of both techniques while compensating each other's limitations [37]. The schematic of such a combined nanoimprint and photolithography (CNP) technique is shown in figure 6. A hybrid mask concept is introduced in this process (shown in figure $6(a)$ ), which is made of UV transparent material and acts both as a NIL mould and as a photolithography mask. Protrusions are made on the mould for imprinting nanoscale features, while metal pads are embedded into the mould serving, as a light mask for photolithography for replicating large patterns. The fabrication details for such a hybrid mould can be found in [38]. The resist used in this method can be either a negative tone photoresist or a UV-curable material.

The processing steps of CNP are very simple. The hybrid mould is first imprinted into the resist layer using pressure, and then the whole mould-substrate assembly is exposed to UV radiation (figure $6(b)$ ). After the hybrid mould and the substrate are separated (figure $6(c)$ ), the substrate is immersed in a developer solution to remove the unexposed resist that is blocked by the metal pads (figure $6(d)$ ). After developing, both large and nanoscale patterns are created in the polymer resist in one step.

To demonstrate its effectiveness in replicating complex patterns, we compared the results of pattern replication using conventional NIL and the new CNP method. A mould that contains both large size patterns (e.g. $200 \mu \mathrm{m}$ and larger) and $350 \mathrm{~nm}$ line width and spacing gratings was fabricated for this

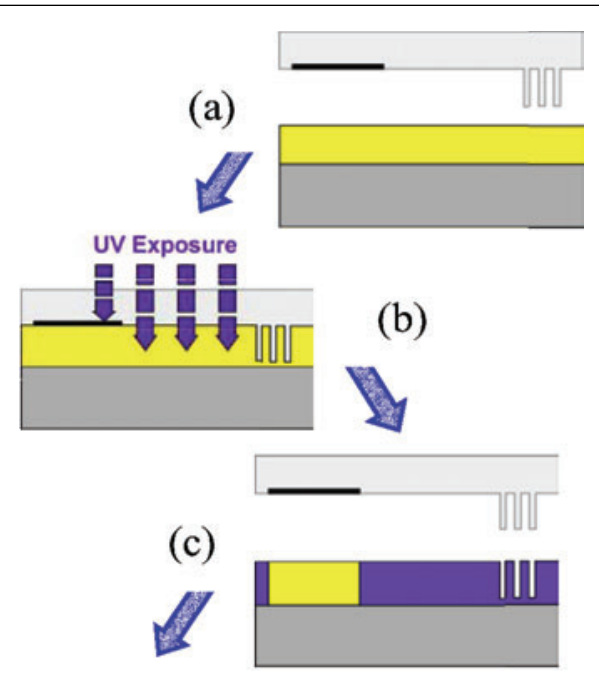

After Developping

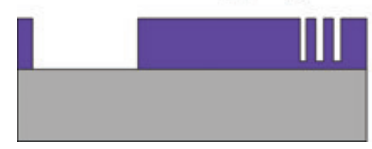

(d)

Figure 6. Schematic of the CNP technique using a hybrid mould (a)
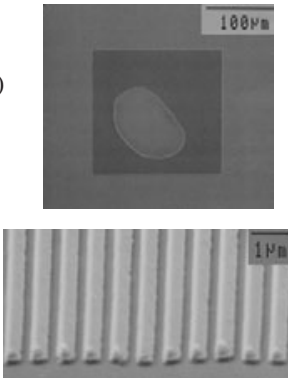

(b)

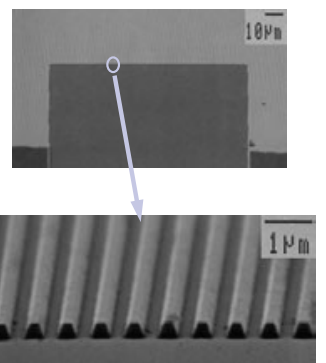

Figure 7. SEM of replicated patterns produced using $(a)$ conventional NIL and $(b)$ the CNP method. Reprinted with permission from Elsevier [37].

purpose. A common negative tone photoresist, SU-8, was used in the experiment. In both cases, the imprinting was done at $80^{\circ} \mathrm{C}$ under a pressure of $50 \mathrm{~kg} \mathrm{~cm}^{-2}$. In the CNP method, the mould-substrate assembly is exposed to a $365 \mathrm{~nm}$ UV light right after imprinting. The mould and the substrate are then separated after baking at $80^{\circ} \mathrm{C}$ on a hotplate for $1 \mathrm{~min}$. The substrate is developed in SU-8 developer solvent for $1 \mathrm{~min}$ to remove unexposed resists underneath the metal pads.

Figure 7(a) shows the SEM images of SU-8 patterns obtained using the conventional NIL technique. It can be seen clearly that every $200 \mu \mathrm{m}$ square has a large void defect in the middle of the resist pattern, which is due to insufficient SU-8 flow during NIL. Also, because the SU-8 melt is not displaced easily, the $350 \mathrm{~nm}$ beam protrusion features on the mould cannot penetrate completely into the SU-8 resist film, leading to a grating pattern that is much shallower than that on the mould. In strong contrast, all these problems are solved in the CNP approach. Figure 7(b) shows the same pattern obtained using the CNP method under the same imprinting conditions followed by a UV exposure and resist developing. No defects were observed in any of the $200 \mu \mathrm{m}$ square patterns. The $350 \mathrm{~nm}$ line pattern was well replicated and has the same height 
as the protrusions on the hybrid mask-mould. From these comparisons, it is clear that the new CNP scheme using a hybrid mould can address effectively the issues in conventional NIL.

There are many advantages to the CNP method using the new hybrid mask concept. Firstly, it enables onestep lithography of arbitrary patterns containing both largescale and nanoscale structures, which is often required in functional device fabrications. Secondly, because there are only nanoscale mould protrusion features on the hybrid mould, it allows a low imprinting pressure to be used since only a very small amount of polymer needs to be displaced. Thirdly, by forming the large patterns as a photomask (i.e. making them as metal pads), it reduces the complexity of the relief pattern on the hybrid mould. This simplifies the residual layer thickness distribution, which can ease the step for residue removal significantly. Finally the throughput could also improve because in NIL imprinting nanoscale features can be a very fast process, while establishing a quasi-equilibrium distribution of residual polymers when imprinting large patterns could take significant time. The last point can be quantified using a simple model by considering the squeezed flow of a Newtonian fluid between plates that have a radius $R$ and a gap distance of $d$. Such a structure can approximate the process where a mould protrusion with size $R$ is imprinted into a resist of thickness $d$ on a substrate. One can obtain a simple solution that expresses the pressure $(P)$ in terms of these dimensions and to the speed of imprinting $(\mathrm{d} h / \mathrm{d} t)$ and fluid viscosity, $\eta: P=\left(3 \eta R^{2} / 4 d^{3}\right)(\mathrm{d} h / \mathrm{d} t)$. Numerical simulation based on finite difference method using the non-steady-state NavierStokes equation has also given such a relationship for simple periodic mould features [39]. Integrating this equation can give the time required to reduce the thickness of the fluidic layer by half (i.e. to imprint halfway through the feature height): $\tau=9 \eta R^{2} / 16 P d^{2}$. This simple model says that under the same pressure the imprinting a time scales as a quadratic function of the pattern size. Therefore, the time required for imprinting a large size pattern will be significantly longer than that for nanopatterns.

A further improvement can be made in this new CNP method, which is to place a metal layer on top of the mould protrusion features to prevent the exposure of the resist layer underneath, so that the unexposed residual layer can be removed easily in a developer solution. This way, it can eliminate the separate residual removal step in NIL completely and could solve simultaneously the non-uniform residual layer thickness problem altogether [40]. Figure 8(a) shows the hybrid mould consisting of $350 \mathrm{~nm}$ line width gratings created in fused silica and the grating top covered with a thin Ni film (that was used as the etch mask when making this mould and therefore does not add complexity to mould fabrication). The mould has been treated with $\mathrm{O}_{2}$ plasma to create a native oxide layer to facilitate silane surfactant coating. We used such a mould to perform both conventional NIL and CNP on negative tone photoresist SU-8. The results are shown in figures $8(b)$ and $(c)$, respectively. It is clear that the CNP method using such a hybrid mould can achieve resist patterns without a residual layer, and the resist patterns can have a higher aspect ratio than the feature on the mould (figure $8(c)$ ). A further benefit of this technique is that the photoresist used can have a better formulation to provide higher etching durability as compared
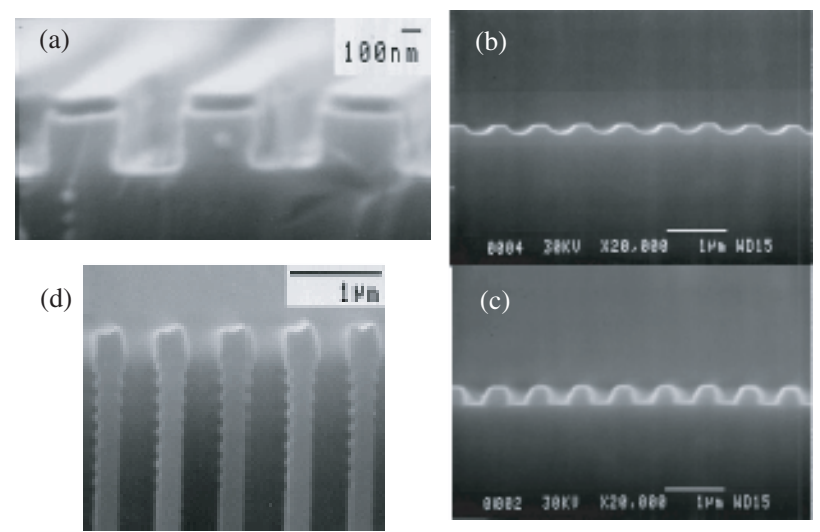

Figure 8. CNP with hybrid mould simplifies imprinting. SEM micrograph of (a) grating hybrid mould showing metal layer on top of protrusion, and SU-8 grating pattern formed using conventional NIL $(b)$ and using CNP technique $(c) ;(d)$ deep trench etching of silicon with cured SU-8 as etch mask.

with thermal plastic polymers that are commonly used in NIL. Figure $8(d)$ shows a SEM of a deep etched Si substrate obtained using the sample shown in figure $8(c)$ and the patterned SU-8 resist as the mask, and over $10 \mu \mathrm{m}$ deep narrow trenches were obtained using a very thin SU-8 resist.

Though there is no apparent resolution limit for NIL due to its purely mechanical embossing nature, the use of light exposure in the CNP and the S-FIL method for UV curing deserves some special consideration because of the possible light diffraction effect. Hirai et al [41] have simulated the light intensity distribution during UV curing exposure in S-FIL and have observed an interesting diffraction and interference effect that could affect the proper curing of the residual polymer layer. In the case of CNP, where a light-blocking layer is placed on top of the protrusions, the light diffraction effect should also be taken into account. One of the reasons why this approach is capable of nanoscale patterning while not suffering from the severe light diffraction effect encountered in conventional photolithography is that the thickness of the residual resist layer under the metal blocking-layer is usually under $100 \mathrm{~nm}$ and the near-field optical effect guarantees that no substantial light scattering or severe proximity effect occurs. It is constructive to compare this method with several lithographic schemes that have been investigated in the past for enhancing the resolution of conventional contact photolithography. These techniques include the use of a metal-embedded mask (MEM), lightcoupling mask (LCM) or traditional metal-protrusion mask (MPM). These schemes use either the optical near-field effect $[42,43]$ or the light coupling technique [44] for resolution enhancement. In all these previous techniques, the resolution is limited by the thickness of the resist layer due to either light diffraction or near-field attenuation. But with the CNP approach, because the mould protrusion penetrates into the resist layer to create the desired resist patterns, the metal layer only needs to block the very thin resist underneath (i.e. the residual layer) to be exposed by light. Therefore the resolution can be greatly enhanced compared with the MEM and the LCM technique because the effective resist layer for CNP is the residual resist layer, which can be controlled to less than tens of nanometres thick, much thinner than the original resist thickness. This ultra-thin resist minimizes effectively the light 

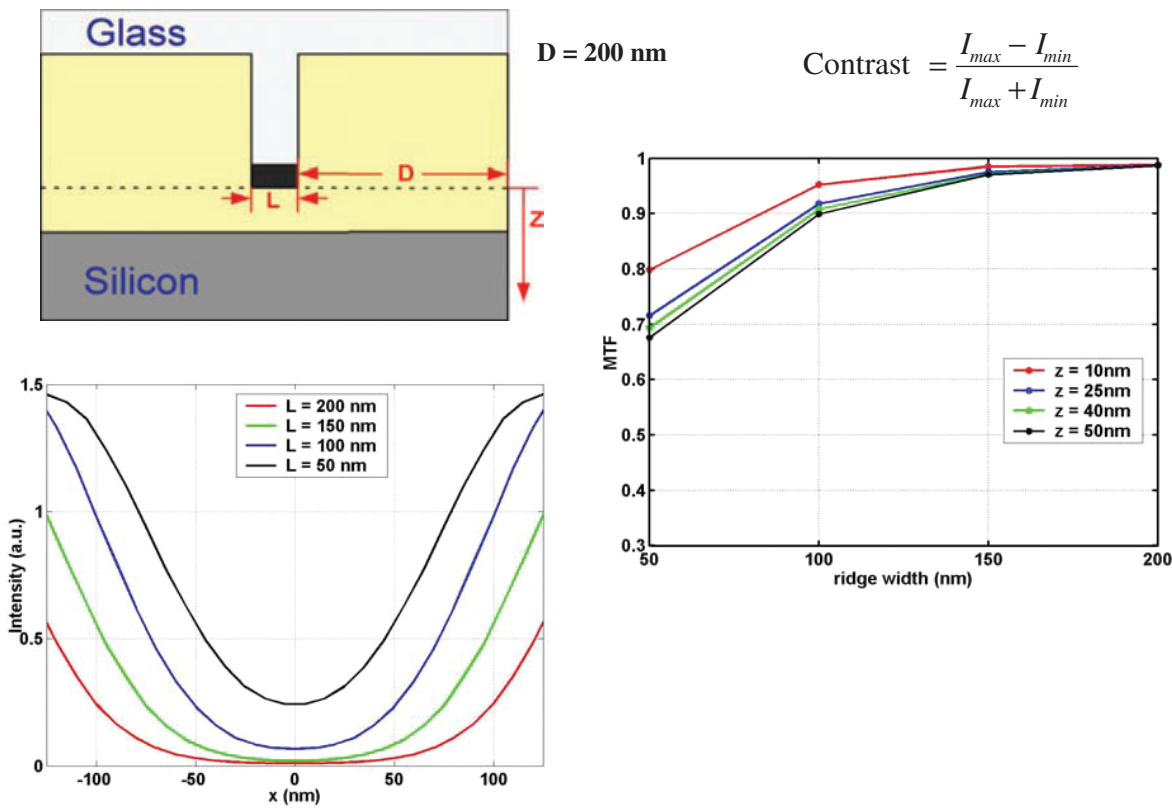

to $50 \mathrm{~nm}$.

diffraction by the metal mask, leading to greatly improved resolution

In order to understand the effect of light diffraction on the resolution limit of this new technique, simulation using the finite difference time domain (FDTD) method was carried out to study the light propagation in a hybrid mould when it is imprinted into a polymer layer under UV exposure. In the simulation, the residue layer is assumed to be $50 \mathrm{~nm}$ thick, with a refractive index of 1.6. The nickel film thickness is also $50 \mathrm{~nm}$, and its refractive index and extinction coefficient are 1.62 and 2.17 , respectively, at a $365 \mathrm{~nm}$ wavelength. Light intensity contrasts are calculated for isolated line patterns with a line width down to $50 \mathrm{~nm}$. Calculation shows that despite the light diffraction effect, a contrast of greater than 0.8 can still be obtained for a $50 \mathrm{~nm}$ line width (figure 9). Therefore, by using a sensitive resist, it should be possible to obtain feature sizes to below $50 \mathrm{~nm}$, much beyond the light diffraction limit.

In summary, the CNP scheme retains most of the advantages of the conventional NIL and S-FIL, such as a low cost, a high throughput and simple processing. In addition, the metal layer on the hybrid mould used in CNP serves as better alignment marks compared with the only relief structures used in S-FIL or NIL because the latter are not able to create much index contrast upon intimate contact with a polymer layer that has a refractive index very close to the polymer resist. Furthermore, a UV-curable liquid resist can be used in combination with CNP to achieve room temperature and low pressure patterning.

\section{Reverse-nanoimprinting technique}

A great advantage of NIL is that it can imprint device structures directly in polymer materials [45] (sometimes in a single step) and create patterned features using polymers with certain desirable physical or chemical functionalities [46-48]. But NIL patterning is typically carried out at a temperature $70-80^{\circ} \mathrm{C}$ above the glass transition temperature $\left(T_{\mathrm{g}}\right)$ of the polymer material to ensure the polymer has a sufficiently reduced viscosity that can be imprinted at a reasonable pressure. For micro- and nanoscale patterning, especially when directly patterning functional polymers, it is often desirable to operate at a lower temperature and with reduced pressure. This is not only required in certain applications or limited by certain substrate characteristics; but if the mould and substrate are made from different materials, with a mismatch in thermal expansion coefficients, a higher temperature will create more stress during the NIL thermal cycle and could present a problem for mask-sample alignment. To target these problems, room temperature NIL has been demonstrated previously using spin-on-glass or hydrogen silsequioxane (HSQ) as the imprint resist material [49, 50], but the pressure needed was very high because of the large modulus of these inorganic materials.

We developed a different approach to this problem based on the following consideration. If a polymer film is spuncoated onto a mould, the polymer will fill up the trench regions of the surface relief patterns. This means that a replica of the mould pattern is formed in the polymer film simply by spincoating. Now if this film can be transferred from the mould to a substrate, patterned structures are obtained. This process functions in the same manner as a lithography technique, and we term it reverse nanoimprinting (figure 10(a)). We have applied reverse nanoimprinting to a few thermal plastic materials such as PS, polycarbonate and PMMA and obtained good pattern results. Figure $10(b)$ shows the imprinted $350 \mathrm{~nm}$ line spacing PMMA grating obtained using the reverse imprint process that was done at a temperature of $105^{\circ} \mathrm{C}$, i.e. at the $T_{\mathrm{g}}$ of PMMA. The key to the successful film transfer lies in the fact that the mould has a lower surface energy than does the substrate, and so the polymer film has better adhesion to the substrate and therefore can be detached from the mould.

Because in reverse imprinting coating of a polymer resist on the substrate is not required, it is therefore possible to use 
this technique to transfer patterns onto substrates that are not suitable for spin-coating or have surface topographies. We have demonstrated that this technique can be applied to flexible substrates and substrates that already have other fabricated features (e.g. a non-flat surface or a pre-patterned surface) to create nanoscale patterns (figure 11) [51]. The ability to pattern over topography has solved a long-standing problem in imprint-based lithography. Previous efforts to solve this problem have often involved multi-layer resist approaches with a thick polymer planarization layer on top of the non-flat substrate [52]. These approaches not only require complex processes with multiple steps but also entail deep etching steps to etch through the thick planarization layer, which often
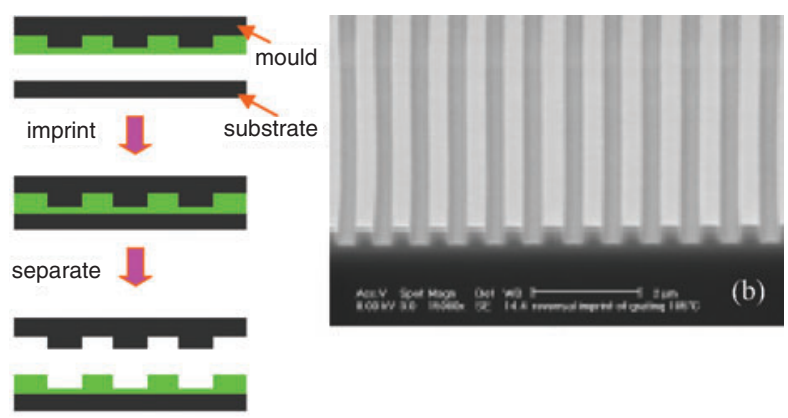

(a)

Figure 10. Schematic of reverse nanoimprint (a), and SEM of reverse imprinted PMMA gratings with $350 \mathrm{~nm}$ line width/spacing $(b)$. Reprinted with permission from the American Vacuum Society [53]. degrades the resolution and fidelity of the pattern. But reverseimprinting has solved this problem very effectively. Figure 12 shows the reverse-imprinted polycarbonate grating structure that is suspended over etched features on a $\mathrm{Si}$ substrate. Such structures can find potential applications where optical elements (e.g. gratings) can be integrated with microfluidic channels for chemical and biological analysis. It is also possible to leave only the imprinted polymer features on top of the underlying structures, as shown in figure $12(b)$, by using more brittle polymers (e.g. PMMA with $M_{\mathrm{w}}$ of 15000 ) that tend to break during mould separation. Such structures can be used as distributed feedback gratings on optical waveguides.

\section{Multi-level patterning of polymer nanostructures}

The reverse-imprinting technique can potentially offer a simple method of fabricating three-dimensional polymer nanostructures. Such three-dimensional structures can be achieved by simply repeating the process and building up the structure in a layer-by-layer fashion (figure 13(a)). Figure 13(b) shows an example of an imprinted threelayer nanostructure, using three different polymers with progressively lower $T_{\mathrm{g}}$ [51]. Note that after imprinting each polymer layer, a dry etching step was used in order to clear the residual polymer layer. To simplify this process, one can incorporate the hybrid mould and CNP concept into this approach by placing a thin metal layer on top of the mould protrusions and by using a UV-curable polymer as the resist. In this case, the residual polymer film after each reverse-imprint can be removed simply by using a developer solution. Another
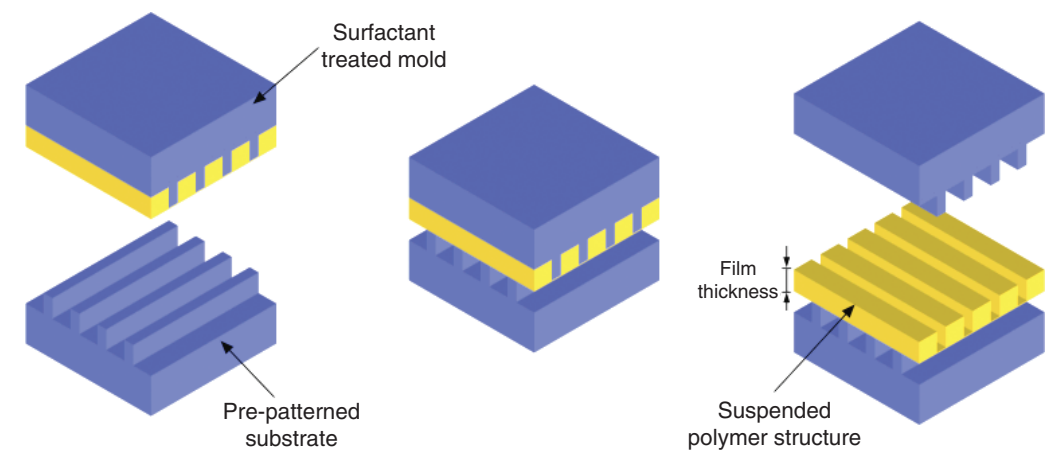

Figure 11. Schematics of reverse-imprinting polymer nanostructures over topography. Reprinted with permission from the American Vacuum Society [51].

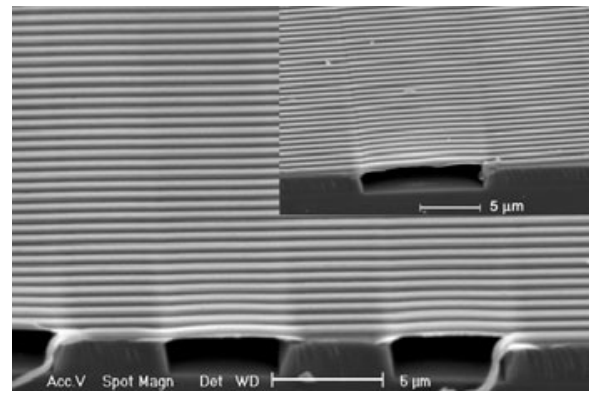

(a)

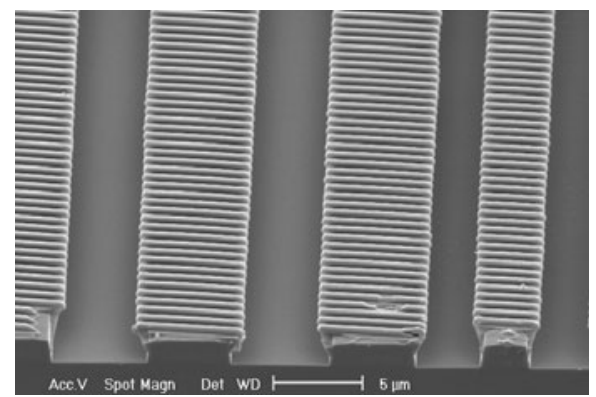

(b)

Figure 12. Imprinting of $700 \mathrm{~nm}$ period grating on topographies: (a) PC pattern imprinted across $5 \mu \mathrm{m}$ gaps and $10 \mu \mathrm{m}$ gaps as shown in the inset. (b) PMMA pattern imprinted on the protruded surfaces for a line spacing greater than $3 \mu \mathrm{m}$. Reprinted with permission from the American Vacuum Society [51]. 


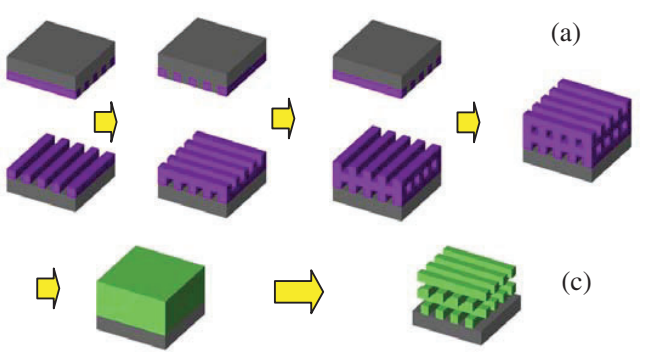

(b)

Figure 13. Schematic of (a) building three-dimensional polymer nanostructures by using reverse-imprinting; thermal plastic or photosensitive material is spin-coated on the mould for pattern transfer. $(b)$ A three-layer polymer nanostructure created by replacing the reverse-imprint process three times. Reprinted with permission from the American Vacuum Society [51]. (c) Infiltrate the three-dimensional periodic structure with other materials, such as inorganic materials that have a high refractive index; then remove the polymer template layer to create a three-dimensional pattern of the infiltrated material that is complementary to the original polymer resist pattern.
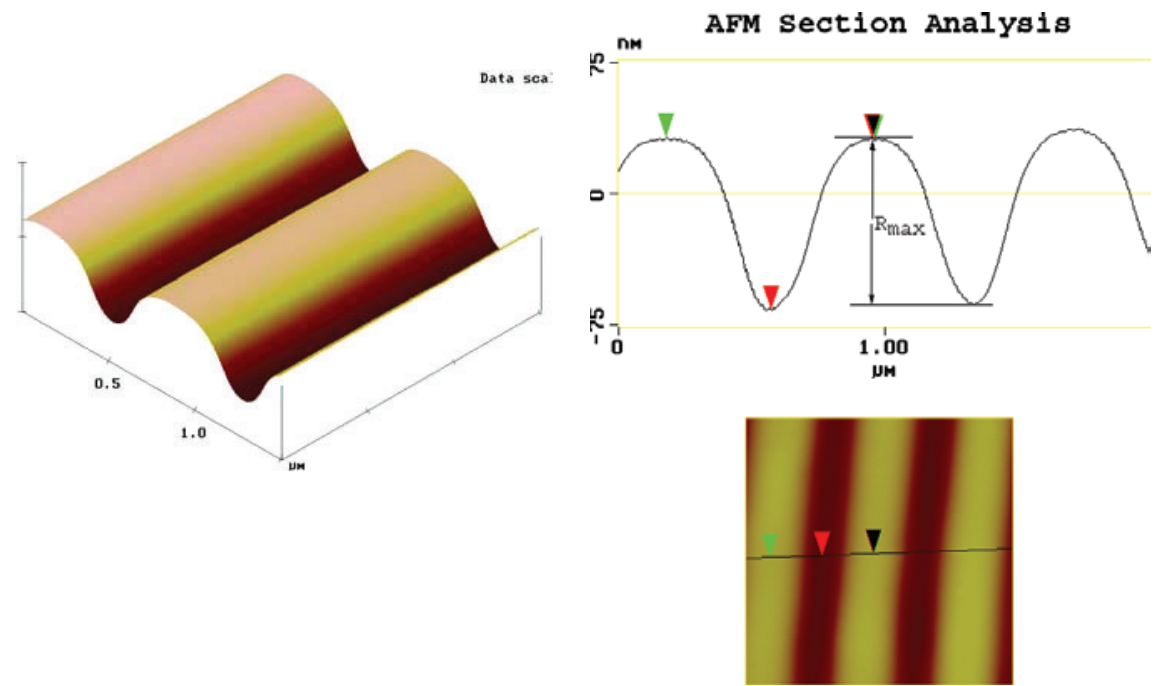

Figure 14. AFM image of a polymer-coated Si mould, with large surface undulations shown by the line scan.

advantage of this approach is that it allows a single polymer material to be used for all layers.

There are many potential applications of such threedimensional polymer nanostructures. A multi-layered structure with varied grating periods can be used as a sizecontrolled filter in microfluidics for selecting and separating particles of different sizes. Another potential application is to fabricate periodic three-dimensional polymer structures and infiltrate the polymer template with high refractive index inorganic materials to create three-dimensional photonic crystal materials (figure 13(c)). Not only can the dimensions of such a structure be designed and controlled, but it is also straightforward to form defect waveguide and resonator structures that are useful for many photonic device applications, by simply removing rows on the mould. This example only serves as an illustration of a potential application of the three-dimensional printing method-we should not underestimate the challenge of stringent pattern alignment required in this particular application.

\section{Polymer inking technique}

A different mode of pattern transfer can occur during reverseimprinting if the mould feature has a high aspect ratio. In this case, the spin-coated polymer film cannot planarize the mould surface, leaving a large undulation on the coated polymer surface (figure 14). When such a polymer-coated mould is brought into contact with a substrate under an applied pressure and at a temperature close to or lower than the $T_{\mathrm{g}}$ of the polymer, only the polymer layer on top of the protrusion is transferred to the substrate, i.e. a different tone can be achieved compared with NIL. Since the process is analogous to stamping with liquid ink, we named this process polymer inking. We have been able to correlate the mode of operation with the imprinting temperature and the degree of planarization from spin-coating [53]. With a suitable degree of surface planarization, successful pattern transfer can be achieved at temperatures and pressures as low as $30^{\circ} \mathrm{C}$ below $T_{\mathrm{g}}$ and $1 \mathrm{MPa}$ in the inking mode [53]. This is a significant advantage over the conventional NIL, which requires an imprinting temperature well above $T_{\mathrm{g}}$ and a very high pressure. Moreover, since relatively little movement of the polymer is involved in the inking mode, it is less sensitive to problems associated with polymer flow.

It is clear from figure 15 that the inked polymer pattern is far from perfect. Most noticeable are the ragged pattern edges (figure 15(b)), which are due to the tearing of the polymer film upon mould separation. It is desirable that the polymer film breaks by itself at the edges of the mould protrusions instead of 
(a)

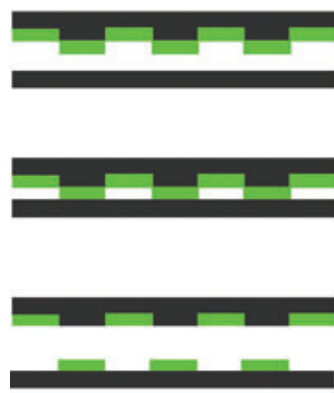

(b)

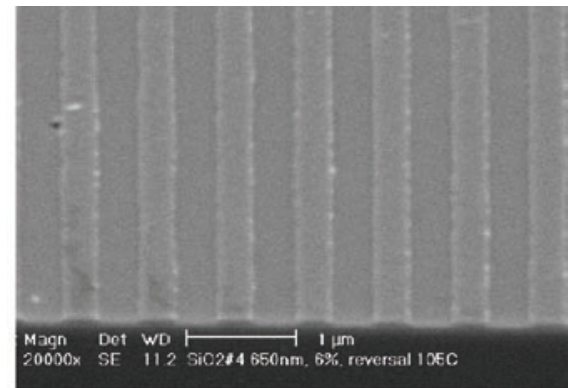

Figure 15. (a) Schematic of polymer inking process. (b) Inked PMMA grating pattern at $105^{\circ} \mathrm{C}$. Reprinted with permission from the American Vacuum Society [53].

(a)

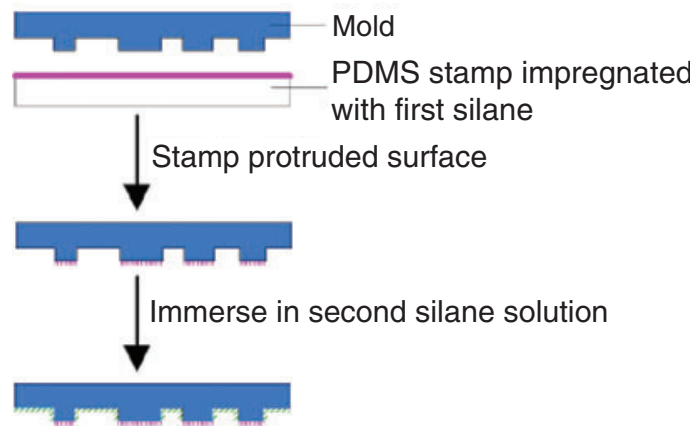

(b)

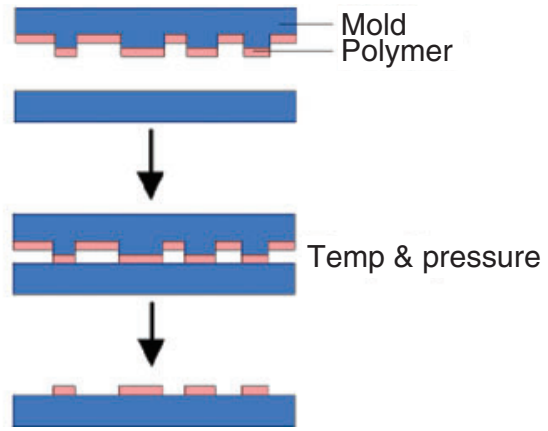

Figure 16. Schematics of $(a)$ selective surface treatment to create discontinuous polymer film on mould, and $(b)$ polymer inking process. Reproduced with permission from the American Vacuum Society [55].

by forced tearing. When it is inked onto the substrate, smoother line edges can be obtained. One way of achieving this goal is to use a selective surface treatment method in which the protruded surfaces and the trenches of the mould are treated to have different surface energies. The difference in surface energy should promote polymer de-wetting near the sidewalls of a feature. This principle is illustrated by the following experiment. A medium surface energy silane molecule (e.g. phenethyl-trichlorosilane (PETS)) is applied to the protruded surfaces of the mould by contacting the mould with a flat PDMS stamp impregnated with such a silane. This step is similar to the PDMS stamping of silane, which will leave a monolayer of covalently linked silane molecules on the protruded surfaces of a $\mathrm{Si}$ or $\mathrm{SiO}_{2}$ mould [54]. Next, the mould is immersed in a solution of another silane molecules with a lower surface energy (e.g. FDTS). Because the protruded surfaces have been silanized with the first silane monolayer, the second silane molecules would form a monolayer only in the recessed trench regions during the immersion (figure 16). Different surface properties can then be achieved for the protruded surfaces and trenches. It was observed that the low surface energy of the trenches causes a polymer thin film to de-wet easily from the sidewalls when the polymer-coated mould was heated to above $T_{\mathrm{g}}$, and the film on the protrusions de-wets to a lesser degree due to the relatively high surface energy. Therefore, the polymer film becomes discontinuous along the edges of the patterns. As a result, the inked polymer onto the substrate has much smoother edges (figure 17).

AFM imaging of the polymer coated mould upon heat treatment also confirmed the de-wetting of the film, as shown in figure 18. For certain polymer materials and deeper mould structures, the polymer can de-wet spontaneously at the

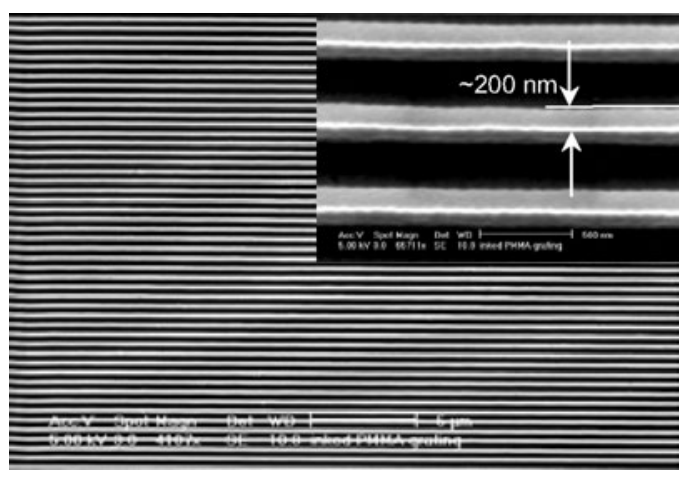

Figure 17. SEM image of inked polymer gratings with $200 \mathrm{~nm}$ line width and smooth edges, achieved at $105^{\circ} \mathrm{C}$ with a selectively treated mould (insert shows a zoom-in perspective view of the inked grating.

sidewalls after spin-coating, leaving isolated polymer islands on top of the protrusions (figure 18(a)). However, it is also clear that the polymer film retracts from the edge of the protrusion. The degree of retraction tends to increase upon heat treatment (figure 18(b)) [55]. Polymer retraction on top of the protrusions implies that the inked pattern will have a lateral size that is different from that of the mould. Although such dimensional shrinkage creates additional issues for faithful pattern transfer; on the other hand, it allows one to obtain polymer patterns with a feature size significantly smaller than that of the mould, which offers a viable approach to obtaining submicrometre features using a mould with a much larger feature size. Our experiments show that a lateral shrinkage as large as $70 \%$ for polycarbonate can be obtained.

We have also developed another method for inking polymers, which is by placing the polymer 'ink' directly on 
(a)

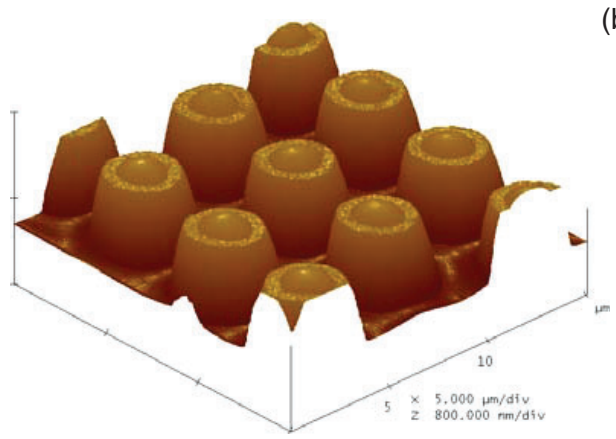

(b)

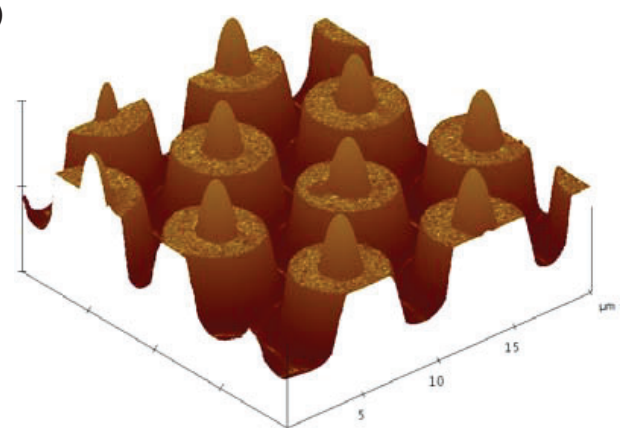

Figure 18. AFM image of PC de-wetting on selectively treated mould surface (a) after spin-coating (Reproduced with permission from the American Vacuum Society [55].) and (b) after annealing at $170^{\circ} \mathrm{C}$.

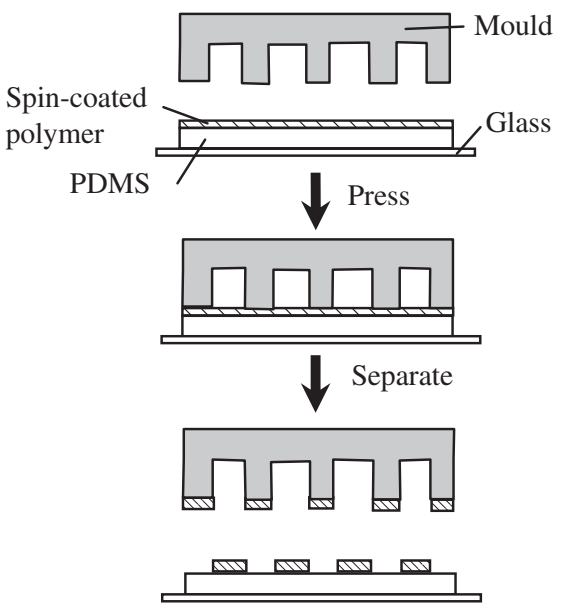

Figure 19. Schematic of inking process using a PDMS ink pad.

top of the mould protrusions. This is achieved by stamping the mould with a special 'ink pad'. Such an ink pad is made of a flat Si mould or a soft PDMS, and coated with a thin polymer layer. The use of an elastomeric pad such as PDMS is found to give better patterning results. The schematic of such an inking process is shown in figure 19. First the polymer to be patterned is spin-coated on a flat $\mathrm{Si}$ mould or a PDMS elastomer that is backed with a hard substrate. A brief $\mathrm{O}_{2}$ plasma treatment of the PDMS is often necessary to create a hydrophilic surface, which ensures a uniform coating of a polar polymer material. Next a Si mould with predefined patterns is pressed against the polymer layer on the ink pad at a temperature close to the $T_{\mathrm{g}}$ of the polymer. Pressing with the mould causes deformation of the polymer layer and the PDMS underlayer if such an elastomeric pad is used and induces a large stress field in the polymer layer near the edge of the mould protrusions. Such stress can lead to a local rupture of the polymer along the protrusion edges. When the mould is removed subsequently from the ink pad, it will take with it the polymer 'ink' that is adhered on top of the protrusion patterns. To enhance the surface energy contrast between the mould and the PDMS for a successful 'inking', the mould is treated usually with a medium energy silane surfactant such as PETS. A preferential adhesion of the polymer on the mould protrusion is obtained because the $\mathrm{O}_{2}$ plasma-treated PMDS will revert to its low surface energy and hydrophobic state in a short time (a few minutes). The inked polymer on top of the mould protrusion can be transferred onto a substrate with a suitable pressure and at a temperature close to $T_{\mathrm{g}}$. Figure 20 shows the PMMA patterns transferred onto a substrate by 'inking' from a hard $\mathrm{Si}$ ink pad (figure 20(a)) and from a PDMS soft pad (figure 20(b)), respectively.

The polymer inking techniques introduced here have several important advantages compared with other patterning techniques such as $\mu \mathrm{CP}$ and NIL. For example, for pattern transfer, the inking polymer can be chosen to have a higher dry etch resistance than that of a self-assembled monolayer (SAM) or polymer layer. While NIL requires an additional etching step for removing the residual film in the imprinted region, the inking methods discussed above do not leave a residual film layer at all, and therefore the $\mathrm{O}_{2}$ plasma etching step used in NIL or S-FIL can be avoided. This property can be applied to fabricating three-dimensional stacked polymer nanostructures as discussed in the previous section and without using an $\mathrm{O}_{2}$ plasma for removing the residual layers in each step. Furthermore, we foresee that the polymer inking method is potentially useful for direct transfer or patterning of polymer materials that have unique functionalities, such as conductive polymers for organic electronics or biomaterials, because no additional solvent or plasma processing steps are needed. Figure 21 shows such an example of a patterned conductive PEDOT created using the polymer inking method. Due to the high conductivity, such micro- and nanoscale PEDOT patterns can be used as electrodes in high density organic electronic circuits.

\section{Device applications}

The high resolution and high throughput capability of nanoimprinting makes it a very useful technique in many device applications that require precision patterning of large areas of nanoscale structures. NIL was first used to fabricate $\mathrm{Si}$ nanodevices [56]. Since then it has been applied to the fabrication of numerous electrical, optical and magnetic devices. These include hybrid plastic electronics [57], organic TFTs and electronics [58,59], diffractive optical elements [60], waveguide polarizers [61], high resolution OLED pixels [62], NLO polymer nanostructures [47], high density quantized magnetic disks [63] and patterned magnetic media [64]. Multilevel NIL has also been demonstrated in the fabrication of working Si MOSFETs [65], in which the alignment is done by IR imaging through a Si substrate. The mould features 

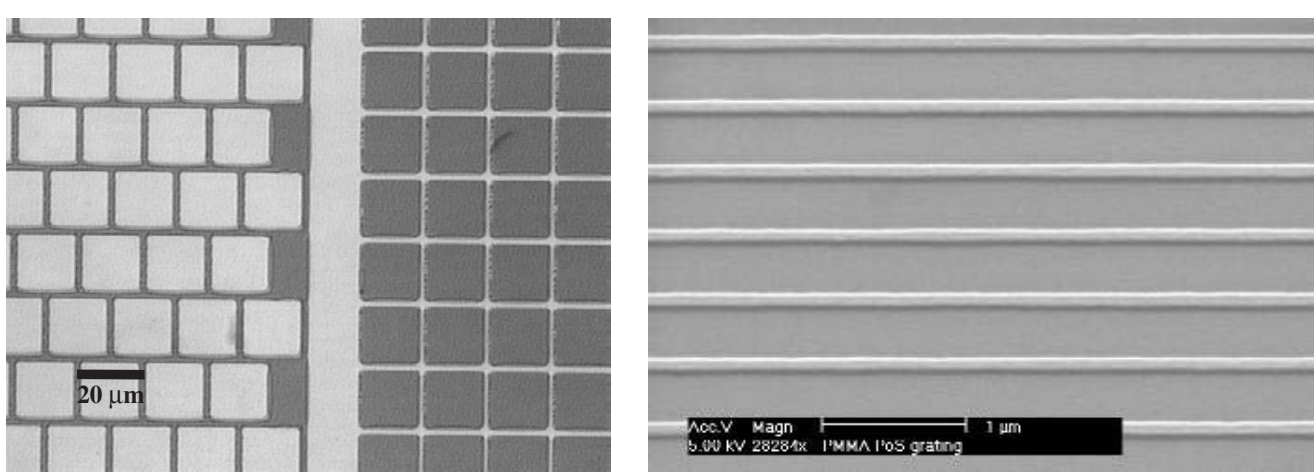

Figure 20. Positive patterns created by further transferring the inked polymer from the mould to a blank wafer at $115^{\circ} \mathrm{C}$ and $50 \mathrm{~kg} \mathrm{~cm}^{-2}$ pressure for $5 \mathrm{~min}$.

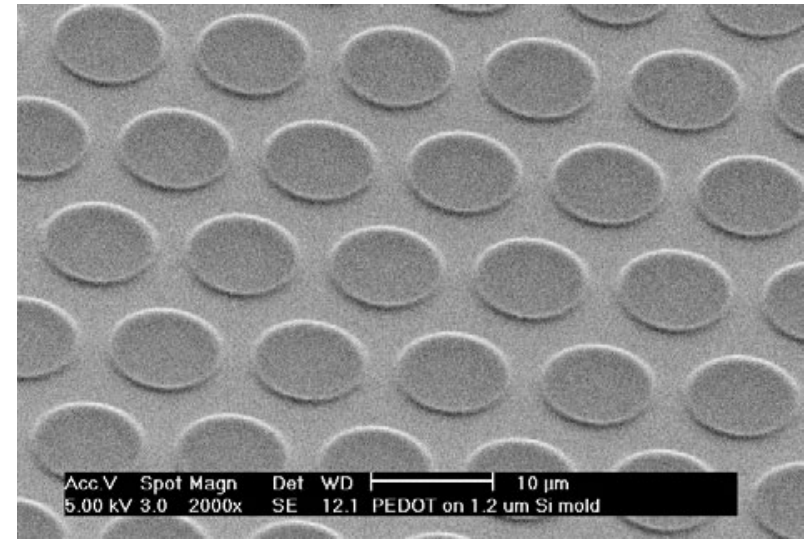

Figure 21. SEM of PEDOT patterns created using the polymer-inking method.

in imprint lithography are not limited to two-dimensional structures but instead could have three-dimensional structures. This is a great strength of the imprint-based technique because it can replicate three-dimensional structures in a single step, while most other techniques multiple steps are involved in achieving the same task. Utilizing this property, threedimensional moulds have been used to fabricate T-gates in HEMT devices [66-68].

Apart from being a lithographic technique for creating resist patterns, NIL can mould structures directly in polymers with nanoscale precision. Many new applications can be exploited from realizing this point. We would like to discuss briefly a few of the applications here and by no means to exhaust the possibilities.

\subsection{Polymer photonic devices}

Because imprinting offers high resolution and high throughput patterning, it is ideally suited for photonic device applications where fabrication of structures involves one critical lithography step. One ideal application is to fabricate twodimensional photonic crystal structures that are made of dielectric materials. Imprint lithography can replace the most commonly used technique, electron-beam lithography, and can pattern large areas of photonic crystal structures in a parallel fashion. The CNP method using a hybrid mould can also allow easy integration of such photonic crystal structures with much larger dielectric waveguides. Other examples are diffractive and sub-wavelength optical elements. Optical quality polymers themselves can be used as materials for building devices. Moreover, the polymer material can be chosen to have certain functionalities.

Imprinting is also suitable for polymer waveguide devices, especially those having certain critical dimensions in the submicron and nanoscale length scales. Towards this end, we have applied NIL to fabrication of polymer micro-ring resonators. A micro-ring resonator is a highly compact photonic device that is typically in the form of a micro-ring coupled closely to a waveguide and offers unique properties such as a narrow bandwidth filtering and a high quality factor. The resonance condition occurs when the round-trip phase acquired by the guided wave in the microring is equal to $2 m \pi$, where $m$ is an integer. The waveguide coupling region is defined by a gap of the order of $100 \mathrm{~nm}$ to ensure sufficient power coupling. Such devices hold great promise for integrated photonics ([69] and reference therein) as well as for highly sensitive detectors for biomolecules [70-72].

Previously, micro-ring resonator devices for optical communication applications have been demonstrated in semiconductor materials using a combination of e-beam lithography and dry etching. We have achieved the fabrication and operation of a polymer micro-ring resonator using a direct imprinting technique (figure 22) [45]. The use of polymeric materials offers a number of advantages over semiconductorbased devices, such as easy film processing and compatibility with different substrates, better coupling to optical fibres and a lower surface roughness scattering, which limits the $Q$-factor of micro-fabricated resonators. The coupling coefficient between the bus waveguide and the microring plays an important role in determining the device characteristics; and it depends exponentially on the gap distance between the ring and the bus waveguide. In order to provide sufficient coupling, the gap width at the coupling region needs to be $100-200 \mathrm{~nm}$. This means that a polymer structure with an aspect ratio of $\sim 10: 1$ is required for such devices. It is very difficult to use a traditional dry etching RIE process to etch a polymer to achieve these stringent dimensions required for proper device operation. But, using imprint lithography, we first fabricated a Si mould that had the reverse patterns of the microring resonators and used this mould to imprint a thin polymer layer (e.g. PS) cast on a substrate (thermal $\mathrm{SiO}_{2}$ on $\mathrm{Si}$ ) under pressure and at a temperature of $175^{\circ} \mathrm{C}$. After cool-down and de-moulding, the residual polymer layer was removed, 

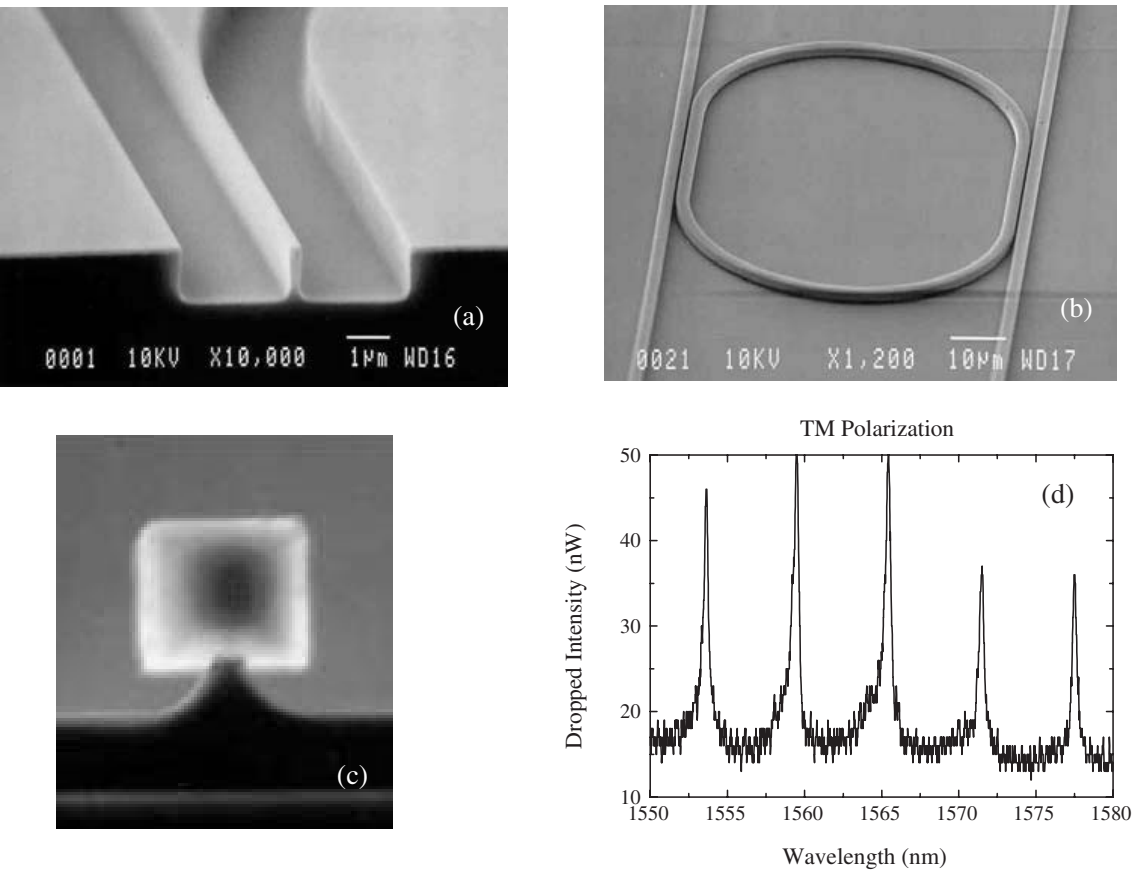

Figure 22. SEM pictures of $(a) \mathrm{SiO}_{2}$ mould used to replicate polymer waveguides. $(b)$ and $(c)$ Perspective view and cross sectional view of polymer micro-ring resonator fabricated using the direct imprinting method. $(d)$ Optical spectrum measured from the drop port of a micro-ring resonator. $(a)$ and $(c)$ reproduced with permission from the American Vacuum Society [45]. $(b)$ and $(d)$ reproduced with permission from the American Institute of Physics [74].

leaving polymer waveguides on top of $\mathrm{SiO}_{2}$ (figure $22(b)$ ). To ensure better mould confinement in the polymer waveguide, we used HF to etch the oxide layer isotropically underneath the waveguide to create a pedestal structure. A cross-section SEM picture of a typical polymer waveguide is shown in figure 22(c). The detailed fabrication process can be found in [45]. Figure 22(d) shows a typical spectrum of the PS microring devices measured from the drop port of the bus waveguide as shown in figure $22(b)$.

Most of the applications of micro-ring devices require a very high $Q$-factor resonance, which is determined by the optical loss factors in the micro-ring cavity. Among the various losses in the micro-ring waveguide, it has been shown through experiments that the surface roughness induced scattering is the dominant loss mechanism in micro-fabricated ring and disk resonators [73]. Surface roughness is a consequence of the fabrication process itself, which has taken care of using special means. Using the polymer flow property at an elevated temperature, we investigated a simple thermal-reflow process for reducing the surface roughness of polymer waveguides [74]. This technique involves heating the polymer devices fabricated with a controlled time duration in a temperature range $10-20^{\circ} \mathrm{C}$ below the glass transition temperature of the polymer. Such heat treatment reduces the viscosity of the material and enhances its fluidity. As a result, the polymer structure can undergo reflow and the surface roughness can reduce significantly under the action of surface tension. A loss reduction in a micro-ring waveguide of over $70 \mathrm{~dB} \mathrm{~cm}^{-1}$ has been achieved using this simple reflow technique [75].

High $Q$-factor resonances can be exploited for optical sensor applications [76, 77]. For biosensor applications, the polymer surface chemical property can be modified easily for specific biomolecule attachment. We conducted experiments for detecting the concentration of glucose in an aqueous solution using such an imprinted PS microring resonator. The change in the concentration of the solution affects both the effective index and the resonant wavelengths, and a linear dependence of the resonance shift versus glucose concentration was observed [71]. By using the imprinting approach, it is possible to 'stamp' out micro-ring-based integrated photonic circuits or arrays of biosensors in a single step, offering practical and scalable solutions to these potential applications.

\subsection{Imprinting structures in functional polymers}

Imprinting can also be applied to polymers that have special functionalities. For example, many NLO polymers offer the potential of a high speed response, great sensitivity and low cost processing for use in advanced optoelectronic systems. NLO polymers have great advantages over their inorganic counterpart because they are easy to process and can be applied to any substrate, which promises direct integration with electronic ICs in a single hybrid optoelectronic package. A fabrication technique that is able to create nanoscale structures in NLO polymers will allow exploitation of a number of interesting phenomena as well as important device applications. The photonic bandgap (PBG) structures described earlier can provide entirely new means of light confinement, guiding and routing using a linear periodic medium [78]. However, it is expected that a non-linear PBG structure offers a much richer and more complex response to light interaction with the periodic NLO media. For instance, control can be exercised over the group and phase velocities of the propagating light beam as well as its field intensity distribution [79]. Applications can be sought that use the enhanced EM wave density of states at the band-edge for 

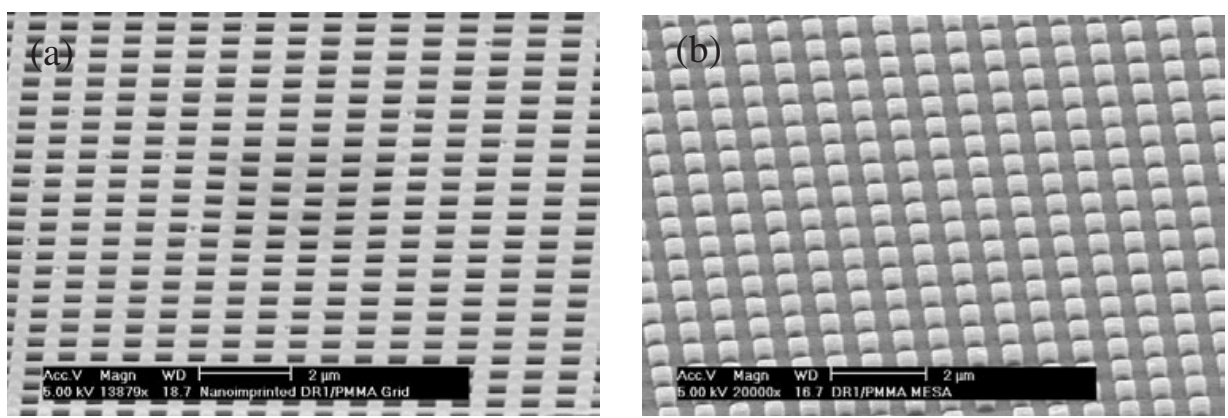

Figure 23. Scanning electron micrographs of nanoimprinted $700 \mathrm{~nm}$ period; (a) two-dimensional air hole arrays in DR1-PMMA; (b) two-dimensional pillar arrays of DR1-PMMA

efficient non-linear interactions between the light field and engineered NLO polymer structures [80]. If the media has Kerr non-linearity, the NLO PBG system will exhibit bistability and multi-stability near the band edges [81], which promises important optical switching applications [82]. To explore these new effects in NLO polymers, it is essential to have a versatile fabrication technique suitable for polymeric materials. Several techniques have been developed in the past for creating optical grating structures [83]. Some of these techniques can also be applied to producing periodic NLO polymer structures, especially in a guest-host system where the origin of optical non-linearity comes from the NLO chromophores doped in a host polymer matrix. For example, photobleaching can be used to create index gratings in a host polymer containing NLO dyes [84]; a periodically modulated poling field can generate spatial modulation of non-linearity [85]; irradiation with two interference laser beams at an absorption wavelength can produce surface relief gratings in NLO dye-doped polymers [86]. However, all these techniques are limited in resolution due to light diffraction or the proximity effect in electric field poling.

We have shown that it is straightforward to apply nanoimprinting to fabricating photonic nanostructures in guest-host NLO polymers [47]. A prefabricated mould can be pressed into a NLO polymer using mechanical force while both the substrate and the mould are held at a temperature above $T_{\mathrm{g}}$. If it is desired that the patterned polymer have a $\chi^{(2)}$ non-linear response, an electric field can be applied at the same time to align the chromophores in the host polymer so as to induce a non-centrosymmetric configuration necessary for secondorder non-linearity. This simultaneous poling feature has been demonstrated by Sugihara et al [87]. After the mould and the substrate are cooled down to room temperature, the mould is separated from the NLO polymer, leaving a complementary pattern in the NLO polymer layer. Creating a PBG structure in a third-order NLO polymer is simpler because the electric poling process can be omitted. Figure 23 shows the SEM micrograph of imprinted periodic structures in a commonly used NLO polymer (PMMA doped with Disperse-Red 1 (DR1) dye [88]). It is expected that many of the guest-host polymeric NLO systems can be patterned in this fashion.

\subsection{Biological applications}

Another area where nanoimprinting-based technology may play an important role is in micro- and nanofluidic devices for biological and lab-on-a-chip applications. Most micro-fluidic devices until now have been fabricated in silica-based materials using standard cleanroom micro-fabrication processes such as photolithography, electron beam lithography, wet etching, reactive ion etching and anodic wafer bonding. Most of these techniques are expensive and not readily accessible to a lot of researchers, especially biologists. To overcome this limitation, low cost and flexible alternatives are highly desirable. Moreover, many applications using inexpensive and disposable devices fabricated on bio-compatible substrates need to be widely acceptable. For instance, biosensors for use in the medical and food examination areas are often required to be disposable, partly due to the fact that enzymes used for enzymatic detection of the analytes are difficult to stabilize over a long lifetime. Point-of-care clinical diagnostics, where cross-contamination is a critical issue, also require disposable assays that can be used cost-effectively in hospitals and clinics. Fabrication techniques based on cheaper and less fragile polymeric materials offer attractive solutions to these requirements. Micro-contact printing and nanoimprinting are two versatile technologies that will likely impact the field of biotechnology (see chapters 13 and 14 in [4]). The imprintingbased method is quite generic since it allows fabrication of structures ranging from micrometres to nanometres in dimension. Furthermore, it can be used to fabricate structures in polymer layers such as fluidic channels and in combination with electrodes or contacts, allowing separation of various types of molecules in combination with detection. Interested readers are referred to chapters 16 and 17 of [4] for more discussions on applying NIL to micro-fluidic and biological applications, where compelling arguments have been made regarding the great potential of NIL in biotechnology.

8.3.1. Nanoscale protein patterning. To illustrate one of the many possibilities, we have demonstrated nanoscale protein patterning based on NIL and surface chemical modification. The biological roles of proteins are extraordinarily diverse and include catalysis, force generation, mechanical support, signalling and sensing. Beyond their central importance to biology, proteins are of great interest because these sub-cellular nanomachines have potential to be integrated into micro- or nanofabricated devices for creating low cost, robust technologies of unprecedented small scale and high efficiency. Applications include biosensors, actuation of micro-electromechanical systems (MEMS) and tissue engineering, as well as screening tools for proteomics 
and pharmacology, and basic biological research $[89,90]$. However, both the study and application of proteins has been challenged by the inherent difficulties associated with positioning these tiny objects. The ability to immobilize biomolecules precisely in well-defined patterns while retaining their native functionality will become a primary enabling technology.

We have developed recently a flexible technique for patterning bioactive proteins selectively with nanoscale resolution using NIL and fluoro-polymer surface passivation, exploiting the specificity of the biotin/streptavidin linkage [91]. This technique achieves high throughput reproducible patterns of biologically active proteins with nanoscale resolution and

Remove PMMA residue with O2 plasma etch

Figure 24. Process flow diagram of substrate patterning and protein adsorption. Spin-coated PMMA polymer is patterned using NIL. Exposed $\mathrm{SiO}_{2}$ regions are etched, and a passivating $\left(\mathrm{CF}_{x}\right)_{n}$ polymer ( $x=1$ or $2, n=$ number of monomer subunits) is deposited during a $\mathrm{CHF}_{3} \mathrm{RIE}$ procedure. Residual PMMA is stripped away with acetone, exposing the underlying $\mathrm{SiO}_{2}$ in the 'patterned regions'. An aminosilane monolayer is attached covalently to the exposed 'patterned regions'. Biotin-succinimidyl ester is then linked covalently to the primary amine of the aminosilane layer, and streptavidin is bound to the biotin layer. Finally, the biotinylated target protein is bound to the streptavidin layer. Reproduced with permission from the American Chemical Society [91] high selectivity. A schematic process flow is shown in figure 24. In our study, patterns of passivated $\mathrm{Si}$ are created using NIL, RIE and plasma deposition, forming a template for patterned chemical modification with an aminosilane monolayer, upon which layers of biotin, streptavidin and ultimately a protein target are bound subsequently. This technique results in highly specific immobilization of a monolayer of biofunctional target protein in patterned areas and less than $0.1 \%$ of a monolayer in surrounding regions. Successful patterning was verified and quantified using epifluorescence microscopy. Fluorescent images of immobilized protein are shown in figure 25 . The most important criterion for any protein patterning technique is specific binding of target proteins, i.e. the technique must produce a high density of biomolecules in desired patterned regions, while preventing non-specific adsorption of these molecules in the unpatterned regions. By using a plasmadeposited fluoropolymer as a passivation material, we have achieved a very high contrast between the patterned proteins and the non-specific adsorbed proteins. Detailed analysis shows a surface density of rhodamine-labelled streptavidin

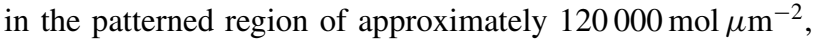
which is of the same order as the surface density expected from a close-packed streptavidin monolayer. Nanopatterned proteins processed using this method can retain their biological functionality, as demonstrated by antigen binding by patterned antibodies (figure 25(c)). This technique is compatible with protein patterning on both oxidized $\mathrm{Si}$ wafers, which may take advantage of the wealth of micro-fabrication techniques developed by the semiconductor industry for integrating immobilized proteins into bio-MEMS devices and optical quality coverglass suitable for microscopic analysis of protein distributions and interactions.

We have demonstrated patterned protein feature sizes down to $75 \mathrm{~nm}$ (figure 26) with high specificity. Since NIL can imprint features of less than $10 \mathrm{~nm}$ across [5], we anticipate that placement of individual biomolecules at designated locations will be possible. The compatibility of this technique with both $\mathrm{SiO}_{2}$ substrates and optical quality cover glass broadens the potential applications of immobilized proteins, allowing easy integration with MEMS and micro-fluidic devices as

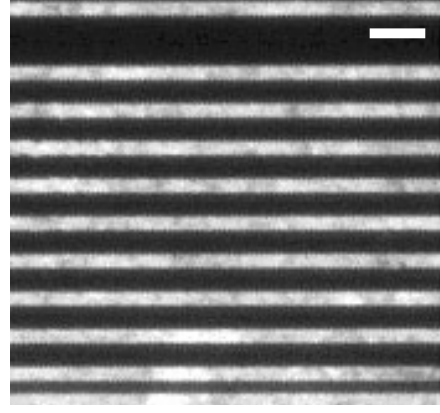

(a)

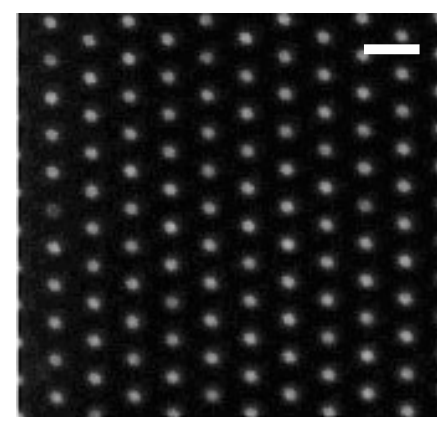

(b)

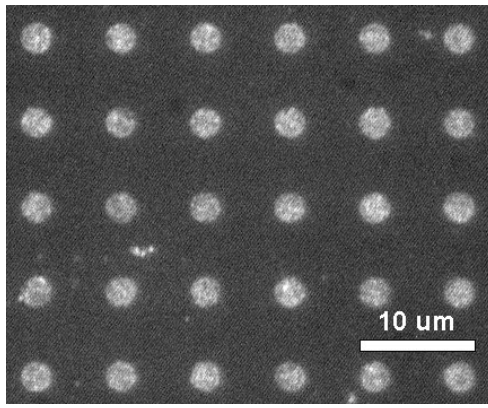

(c)

Figure 25. Epi-fluorescence image of rhodamine-labelled streptavidin bound to uniform $(a)$ micro-scale lines and $(b)$ dots of biotinylated BSA protein on oxidized Si substrates. Fluorescent intensity signal in the passivated regions is at or below the noise level of the imaging system, indicating the fluorophore concentration in these areas is less than $0.1 \%$ of that observed in the patterned regions. The patterned streptavidin density is estimated to be $120000 \mathrm{~mol} \mu \mathrm{m}^{-2}$. (c) Epi-fluorescence image demonstrating the retained biological activity of patterned biomolecules. Biotinylated polyclonal anti-catalase antibody was patterned in $2 \mu \mathrm{m}$ dots, and the antibody's fluorescent antigen, rhodamine-labelled catalase, binds selectively to the antibody-patterned regions. The surface density of the bound catalase is approximately $31000 \mathrm{~mol} \mu \mathrm{m}^{-2}$. The scale bars are $10 \mu \mathrm{m}$ in all three images. Reproduced with permission from the American Chemical Society [91]. 
well as ready access to a wide range of optical imaging, measurement and manipulation methods. Ligand-binding assays based on protein micro-arrays hold great promise for biosensing, investigating proteomics and protein-protein or enzyme-substrate interactions, studying cell adhesion and motility or building immunoassays based on antigen-antibody binding. It is clear that NIL has the potential to become a favourable method for making nanoscale structures for various bio-applications.

\subsubsection{Nanofluidic devices. A second application is} in the micro- and nanofluidics area. Nanofluidics is an emerging field of study that has much technological significance. It is attractive for fundamental study of fluid transport and the molecular behaviour at extremely small dimensions and for high sensitivity single molecule detection $[92,93]$. The interaction of biomolecules (such as DNA) with nanostructured channels having dimensions close to the persistence length of the molecule $(\sim 50 \mathrm{~nm})$ provides a new way of detecting and separating the molecules. Despite the relative ease of constructing nanoscale structures, the sealing of these structures into functional nanofluidic devices presents great technical challenges. Previous sealing methods of constructing nanofluidic channels include anodic bonding of a glass coverslip [94] and soft elastomeric bonding to prefabricated nanochannels on a substrate [95]. While it is possible, the high temperature and the high voltage used in the anodic bonding process will greatly limit its use. On the other hand, the use of a soft elastomer such as PDMS for bonding to nanofluidic channels that have dimensions of $100 \mathrm{~nm}$ or less often results in the partial or complete filling of the channel due to the rubber-like behaviour of elastomeric materials. Sacrificial layer etching using thermal-degradable materials [96] or polysilicon [97] can also be used to form nanofluidic channels. However, the removal of the sacrificial layer in long nanochannels is non-trivial if not impossible. Via holes are often necessary for reducing the time needed to remove the sacrificial layer to a reasonable scale, which increases the device fabrication complexity. Recent progress has demonstrated nanofluidic channels fabrication by using a non-uniform film deposition process such as multiple vacuum evaporation or shadow sputtering [98]. Nonetheless, it is a rather intricate and time-consuming process that requires very careful control of the non-uniform deposition conditions. While this process shows great promise for sealing nanoscale channels, it would present a great challenge for sealing microscale channels in the same step that is often required for interfacing the micro- to the nanoenvironment [99].

We have developed a simple method of fabricating enclosed nanofluidic channels based on a modified imprinting approach [100]. When NIL is used for lithography, the mould protrusions are imprinted into a polymer layer with a thickness that is sufficient for obtaining complete filling of the trenches. This way it ensures a faithful pattern replication. We modified this process slightly for fabricating sealed nanochannels: if a very thin polymer layer is used during imprinting, the displaced polymer will not be able to fill the trenches on the mould completely, therefore creating enclosed nanochannel features (figure 27(a)). The fabrication process can also be well controlled to give a predictable channel width and channel

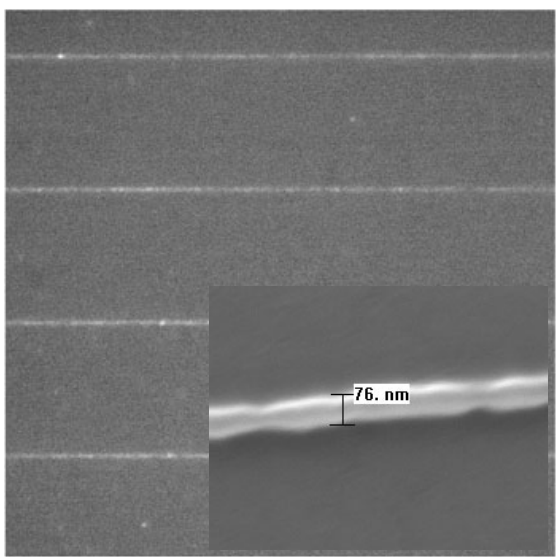

Figure 26. Fluorescent micrograph of biotinylated bovine serum albumin (BSA) bound to the $75 \mathrm{~nm}$ wide lines pattern (SEM of the nanoline patterns are shown in the inset).

height. The channel width is determined by the feature sizes on the channel template used for imprinting. The channel height can be controlled by the depth of the template and the initial thickness of the polymer layer, as well as by adjusting the ratio of the ridge width to the trench width on the channel template. One of the biological applications has been illustrated by stretching genomic DNA in such nanochannel arrays. Over $90 \%$ stretching has been observed in nanochannels with a cross section size of $\sim 120 \mathrm{~nm} \times 75 \mathrm{~nm}$ (figure $27(b)$ and $(c)$ ). Therefore nanofluidic channels could be used for studying the statics as well as the dynamics of DNA molecules in confined geometries [101]. This effect could lead to important biological applications, such as quick mapping of restrictioncut genomic DNA segments in a very short time (minutes), as compared with the hours to days required in conventional pulsed field gel-electrophoresis [102]. It would also reduce the DNA sample amount from nanograms to femtograms (genomic DNA material in a single cell).

The modified imprinting method provides a simple solution for the fabrication of nanofluidic channels. This method can be extended one step further for developing an all-polymer-based nanofluidic channel that can be fabricated solely by the nanoimprinting process, leading to a very low cost and high throughput fabrication of nanofluidic chips. The use of polymer materials for nanofluidic channels also opens up opportunities for exploiting rich polymer chemistry in nanofluidic applications. Patterned arrays of nanofluidic channels may be used for single molecule detection, quick DNA sizing, integrated DNA or protein chips, or high throughput drug screening. These developments may provide a useful fabrication tool for performing genomic, proteomic and chemical analyses at the nanoscale.

\section{Conclusion}

Nanoimprinting technique has, for the first time, enabled a parallel nanoscale processing capability with simple equipment. The simplicity of this method has made it appealing to researchers in various fields. New techniques based on the concept of mechanical printing or embossing are 


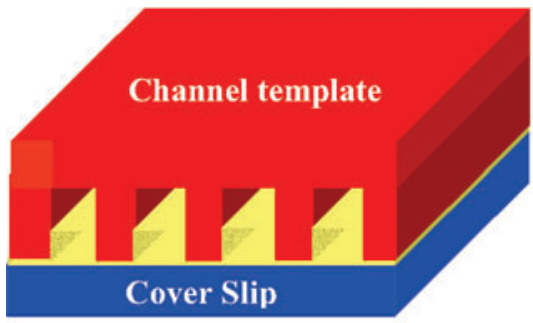

(a)

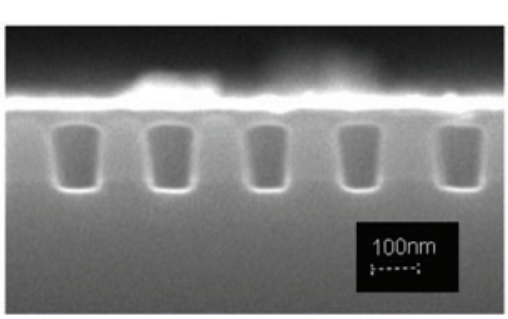

(b)

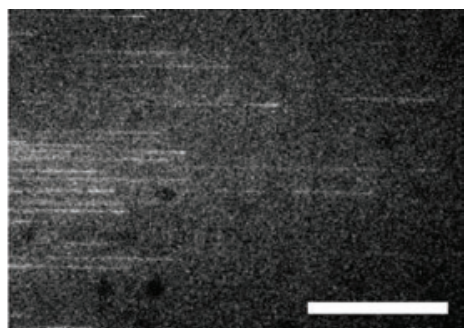

(c)

Figure 27. (a) Schematic showing nanofluidic channel fabrication by using a template mould for imprinting into a thin polymer layer to leave unfilled and self-enclosed channels. (b) SEM micrograph of imprinted nanofluidic channels with cross sections of $75 \mathrm{~nm} \times 120 \mathrm{~nm}$. (c) Fluorescent images showing the stretching of $103 \mathrm{~kb}$ long T5 phage DNA in the nanochannels reaching about $95 \%$ (scale bar $50 \mu \mathrm{m}$ ). Reproduced with permission from the American Chemical Society [91].

appearing very rapidly. More and more researchers are being attracted by these non-conventional patterning technologies due to their scalability, low cost and potential new applications. It is certain that advances in new materials for imprinting will fuel the development in this field. Significant effort and resources are required for investigating the issues involved in enabling imprinting lithography for CMOS fabrication. On the other hand, one can expect more and more nontraditional microelectronics applications to appear in the future years, especially in the areas of photonics, organic electronics and biotechnology. The elimination of high energy photon, electron and ion beams in nanopatterning and the possibility of avoiding dry etching or solvent processing is especially attractive for biological applications. Furthermore, the techniques described in this article also open up the possibilities of creating patterns in multi-layer polymers having different chemical functionalities.

We hope that this review provides the readers with an overview of some aspects of the rapid development of the field of nanoimprinting technology and its various applications in recent years, and it is by no means exhaustive. We wish to inspire more researchers to explore this technique and to reveal many new possibilities in the future.

\section{Acknowledgments}

The author is indebted to all the graduate students and postdoctoral fellows, as well as colleagues during past and current collaborations, at the University of Michigan who have contributed to the work described here: Xing Cheng, ChungYen Chao, Li-Jing Cheng, J Damon Hoff, Drs Li-Rong Bao, Xu-Dong Huang, Tan Li and Yen-Peng Kong and Professors Stella W Pang, Albert F Yee, Alan J Hunt and Chia-Fu Chou (Arizona State University). This review is a dedication to all of them. The author is also grateful for the financial support from AFOSR, ONR, DARPA, NSF and IMRE Singapore that has made this work possible.

\section{References}

[1] Chou S Y, Krauss P R and Renstrom P J 1995 Appl. Phys. Lett. 673114

[2] Chou S Y, Krauss P R and Renstrom P J 1996 Science 27285

[3] Special Report 2003 Technol. Rev. 10636

[4] Sotomayor T S (ed) 2003 Alternative Lithography (Boston: Kluwer)
[5] Chou S Y, Krauss P R, Zhang W, Guo L J and Zhuang L 1997 J. Vac. Sci. Technol. B 152897

[6] Colburn M et al 1999 Proc. SPIE 3676 379-89

[7] Ruchhoeft P et al 1999 J. Vac. Sci. Technol. B 172965

[8] Bailey T C et al 2002 Microelectron. Eng. 61-2 461

[9] Dauksher W J et al 2002 J. Vac. Sci. Technol. B 202857

[10] Bailey T, Choi B J, Colburn M, Meissl M, Shaya S, Ekerdt J G, Sreenivasan S V and Willson C G $2000 \mathrm{~J}$. Vac. Sci. Technol. B 183572

[11] Resnick D J et al 2003 J. Vac. Sci. Technol. B 212624

[12] Li M T 2003 PhD Thesis Princeton University, Princeton

[13] Taniguchi J, Tokano Y, Miyamoto I, Komuro M, Hiroshima H, Kobayashi K, Miyazaki T and Ohyi H 2000 Japan. J. Appl. Phys. Part 1-Regular Papers Short Notes Rev. Papers 397070

[14] Taniguchi J, Tokano Y, Miyamoto I, Komuro M and Hiroshima H 2002 Nanotechnology 13592

[15] Pang S W, Tamamura T, Nakao M, Ozawa A and Masuda H 1998 J. Vac. Sci. Technol. B 161145

[16] Jaszewski R W, Schift H, Schnyder B, Schneuwly A and Groning P 1999 Appl. Surf. Sci. 143301

[17] Ulman A 1991 An Introduction to Ultrathin Organic Films (San Diego: Academic)

[18] Parikh A N, Allara D L, Azouz I B and Rondelez F 1994 J. Phys. Chem. 987577

[19] Beck M, Graczyk M, Maximov I, Sarwe E L, Ling T G I, Keil M and Montelius L 2002 Microelectron. Eng. 61-2 441

[20] Taniguchi J, Kawasaki T, Tokano Y, Kogo Y, Miyamoto I, Komuro M, Hiroshima H, Sakai N and Tada K 2002 Japan. J. Appl. Phys. Part 1-Regular Papers Short Notes Rev. Papers 414194

[21] Yeganeh M S, Dougal S M, Polizzotti R S and Rabinowitz P 1995 Phys. Rev. Lett. 741811

[22] Khang D Y, Kang H, Kim T and Lee H H 2004 Nano Lett. 4633

[23] Khang D Y and Lee H H 2004 Langmuir 202445

[24] Sperling L H 1992 Introduction to Physical Polymer Science (New York: Wiley)

[25] Jung G Y, Ganapathiappan S, Li X, Ohlberg D A A, Olynick D L, Chen Y, Tong W M and Williams R S 2004 Appl. Phys. A:-Mater. Sci. Process. 781169

[26] Khang D Y and Lee H H 2000 Appl. Phys. Lett. 76870

[27] Hirai Y, Fujiwara M, Okuno T, Tanaka Y, Endo M, Irie S, Nakagawa K and Sasago M 2001 J. Vac. Sci. Technol. B 19 2811

[28] Chou S Y, Krauss P R and Renstrom P J 1996 J. Vac. Sci. Technol. B 144129

[29] Khang D Y, Yoon H and Lee H H 2001 Adv. Mater. 13749

[30] Schift H, Heyderman L J, Maur M A D and Gobrecht J 2001 Nanotechnology 12173

[31] Heyderman L J, Schift H, David C, Gobrecht J and Schweizer T 2000 Microelectron. Eng. 54229 
[32] Otto M, Bender M, Hadam B, Spangenberg B and Kurz H 2001 Microelectron. Eng. 57-8 361

[33] Scheer H C, Schulz H, Hoffmann T and Torres C M S 1998 J. Vac. Sci. Technol. B 163917

[34] Scheer H C and Schulz H 2001 Microelectron. Eng. 56311

[35] Pfeiffer K, Fink A, Gruetzner G, Bleidiessel G, Schulz H and Scheer H 2001 Microelectron. Eng. 57-8 381

[36] Montelius L, Heidari B, Graczyk M, Maximov I, Sarwe E L and Ling T G I 2000 Microelectron. Eng. 53521

[37] Cheng X and Guo L J 2004 Microelectron. Eng. 71288

[38] Cheng X, Chang M-H and Guo L J 2004 Proc. SPIE 5374 22-7

[39] Wu L and Chou S Y 2003 47th EIPBN Technical Digest 293-4

[40] Cheng X and Guo L J 2004 Microelectron. Eng. 71277

[41] Hirai Y, Kikuta H and Sanou T 2003 J. Vac. Sci. Technol. B 212777

[42] Kunz R, Rothschild R M and Yeung M S 2003 J. Vac. Sci. Technol. B 2178

[43] Goodberlet J G and Kavak H 2002 Appl. Phys. Lett. 81 1315

[44] Schmid H, Biebuyck H, Michel B and Martin O J F 1998 Appl. Phys. Lett. $\mathbf{7 2} 2379$

[45] Chao C Y and Guo L J 2002 J. Vac. Sci. Technol. B 20 2862

[46] Wang J, Sun X Y, Chen L and Chou S Y 1999 Appl. Phys. Lett. 752767

[47] Guo L J, Cheng X and Chao C Y 2002 J. Mod. Opt. 49663

[48] Pisignano D, Persano L, Visconti P, Cingolani R, Gigli G, Barbarella G and Favaretto L 2003 Appl. Phys. Lett. 83 2545

[49] Matsui S, Igaku Y, Ishigaki H, Fujita J, Ishida M, Ochiai Y, Komuro M and Hiroshima H $2001 \mathrm{~J}$. Vac. Sci. Technol. B 192801

[50] Matsui S, Igaku Y, Ishigaki H, Fujita J, Ishida M, Ochiai Y, Namatsu H and Komuro M 2003 J. Vac. Sci. Technol. B 21688

[51] Bao L R, Cheng X, Huang X D, Guo L J, Pang S W and Yee A F 2002 J. Vac. Sci. Technol. B 202881

[52] Sun X Y, Zhuang L, Zhang W and Chou S Y 1998 J. Vac. Sci. Technol. B 163922

[53] Huang X D, Bao L R, Cheng X, Guo L J, Pang S W and Yee A F 2002 J. Vac. Sci. Technol. B 202872

[54] Jeon N L, Finnie K R, Branshaw K and Nuzzo R G 1997 Langmuir 133382

[55] Bao L R, Tan L, Huang X D, Kong Y P, Guo L J, Pang S W and Yee A F 2003 J. Vac. Sci. Technol. B 212749

[56] Guo L J, Krauss P R and Chou S Y 1997 Appl. Phys. Lett. 71 1881

[57] McAlpine M C, Friedman R S and Lieber D M 2003 Nano Lett. 3443

[58] Austin M D and Chou S Y 2002 Appl. Phys. Lett. 814431

[59] Cedeno C C et al 2002 Microelectron. Eng. 61-2 25

[60] Hirai Y, Harada S, Kikuta H, Tanaka Y, Okano M, Isaka S and Kobayasi M 2002 J. Vac. Sci. Technol. B 202867

[61] Wang J, Schablitsky S, Yu Z N, Wu W and Chou S Y 1999 J. Vac. Sci. Technol. B 172957

[62] Cheng X, Hong Y T, Kanicki J and Guo L J 2002 J. Vac. Sci. Technol. B 202877

[63] Wu W, Cui B, Sun X Y, Zhang W, Zhuang L, Kong L S and Chou S Y 1998 J. Vac. Sci. Technol. B 163825

[64] McClelland G M, Hart M W, Rettner C T, Best M E, Carter K R and Terris B D 2002 Appl. Phys. Lett. 811483

[65] Zhang W and Chou S Y 2001 Appl. Phys. Lett. 79845

[66] Li M T, Chen L and Chou S Y 2001 Appl. Phys. Lett. 783322

[67] Macintyre D S, Chen Y, Lim D and Thoms S 2001 J. Vac. Sci. Technol. B 192797
[68] Chen Y, Macintyre D, Boyd E, Moran D, Thayne I and Thoms S 2002 J. Vac. Sci. Technol. B 202887

[69] Little B E and Chu S T 2000 Opt. Phon. News 1124

[70] Krioukov E, Klunder D J W, Driessen A, Greve J and Otto C 2002 Opt. Lett. 27512

[71] Chao C Y and Guo L J 2003 Appl. Phys. Lett. 831527

[72] Boyd R W and Heebner J E 2001 Appl. Opt. 405742

[73] Van V, Absil P P, Johnson F G, Hryniewicz J V and Ho P T 2001 J. Lightwave Technol. 191734

[74] Chao C-Y and Guo L J 2004 Appl. Phys. Lett. 842479

[75] Chao C-Y and Guo L J 2004 IEEE Photon. Technol. Lett. at press

[76] Vollmer F, Braun D, Libchaber A, Khoshsima M, Teraoka I and Arnold S 2002 Appl. Phys. Lett. 804057

[77] Krioukov E, Greve J and Otto C 2003 Sensors Actuators 9058

[78] Joannopoulos J D, Meade R D and Winn J N 1995 Photonic Crystals: Moulding the Flow of Light (Princeton, NJ: Princeton University Press)

[79] Saltiel S and Kivshar Y S 2000 Opt. Lett. 251204

[80] Scalora M et al 1997 Phys. Rev. A 563166

[81] Li Q, Chan C T, Ho K M and Soukoulis C M 1996 Phys. Rev. B 5315577

[82] Gibbs H M 1985 Optical Bistability: Controlling Light with Light (Orlando, FL: Academic)

[83] Kawatsuki N and Uetsuki M 1992 Polymers for Lightwave and Integrated Optics ed L A Hornak (New York: Mercel Dekker)

[84] Hattori T, Shibata T, Nodera S and Aino T 2000 J. Appl. Phys. 873240

[85] Rikken G L J A, Seppen C J E, Nijhuis S and Meijer E W 1991 Appl. Phys. Lett. 58435

[86] Munakata K, Harada K, Yoshikawa N, Itoh M, Umegaki S and Yataga T 1999 Opt. Rev. 6518

[87] Sugihara O, Nakanishi M, Fujimura H, Egami C and Okamoto N 2000 Opt. Lett. 251028

[88] Kuzyk M G, Sohn J E and Dirk C W 1990 J. Opt. Soc. Am. B 7842

[89] Kane R S, Takayama S, Ostuni E, Ingber D E and Whitesides G M 1999 Biomaterials 202363

[90] Douvas A, Argitis P, Misiakos K, Dimotikali D, Petrou P S and Kakabakos S E 2002 Biosensors Bioelectron. 17269

[91] Hoff J D, Cheng L J, Meyhofer E, Guo L J and Hunt A J 2004 Nano Lett. 4853

[92] Levene M J, Korlach J, Turner S W, Foquet M, Craighead H G and Webb W W 2003 Science 299682

[93] Tegenfeldt J O, Bakajin O, Chou C-F, Chan S, Austin R H, Chan E, Duke T and Cox E C 2001 Phys. Rev. Lett. 86 1378

[94] Stjernstrom M and Roeraade J 1998 J. Micromech. Microeng. 833

[95] Chou H P, Spence C, Scherer A and Quake S 1999 Proc. Natl Acad. Sci. USA 9611

[96] Li W, Tegenfeldt J O, Chen L, Austin R H, Chou S Y, Kohl P A, Krotine J and Sturm J C 2003 Nanotechnology 14578

[97] Turner S W, Perez A M, Lopez A and Craighead H G 1998 J. Vac. Sci. Technol. B 163835

[98] Cao H, Yu Z N, Wang J, Tegenfeldt J O, Austin R H, Chen E, Wu W and Chou S Y 2002 Appl. Phys. Lett. 81174

[99] Cao H, Tegenfeldt J O, Austin R H and Chou S Y 2002 Appl. Phys. Lett. 813058

[100] Guo L J, Cheng X and Chou C F 2004 Nano Lett. 469

[101] Austin R H, Brody J P, Cox E C, Duke T and Volkmuth W 1997 Phys. Today 5032

[102] Lai E 1991 Electrophoresis of Large DNA Molecules: Theory and Applications (New York: Cold Spring Harbor Laboratory Press) 